\title{
Imaging of buried objects from experimental backscattering time dependent measurements using a globally convergent inverse algorithm
}

\author{
Nguyen Trung Thành ${ }^{\diamond}$, Larisa Beilina ${ }^{\circ}$, Michael V. Klibanov ${ }^{\diamond}$ \\ and Michael A. Fiddy ${ }^{\circ}$ \\ ${ }^{\diamond}$ Department of Mathematics \& Statistics, \\ University of North Carolina at Charlotte, USA \\ Emails: tnguy152@uncc.edu, mklibanv@uncc.edu \\ ${ }^{\circ}$ Department of Mathematical Sciences, \\ Chalmers University of Technology and Gothenburg University, Sweden \\ Email: larisa@chalmers.se. \\ ${ }^{\circ}$ Optoelectronics Center, Univeristy of North Carolina at Charlotte, USA. \\ Email: mafiddy@uncc.edu
}

\begin{abstract}
We consider the problem of imaging of objects buried under the ground using backscattering experimental time dependent measurements generated by a single point source or one incident plane wave. In particular, we estimate dielectric constants of those objects using the globally convergent inverse algorithm of Beilina and Klibanov. Our algorithm is tested on experimental data collected using a microwave scattering facility at the University of North Carolina at Charlotte. There are two main challenges working with this type of experimental data: (i) there is a huge misfit between these data and computationally simulated data, and (ii) the signals scattered from the targets may overlap with and be dominated by the reflection from the ground's surface. To overcome these two challenges, we propose new data preprocessing steps to make the experimental data to be approximately the same as the simulated ones, as well as to remove the reflection from the ground's surface. Results of total 25 data sets of both non blind and blind targets indicate a good accuracy.
\end{abstract}

Keywords: Buried object detection, coefficient identification problems, wave equation, globally convergent algorithm, experimental data, data preprocessing.

AMS classification codes: 35R30, 35L05, 78A46.

\section{Introduction}

In 44 a globally convergent algorithm for a coefficient inverse problem (CIP) for a hyperbolic equation was proposed. Since then several follow-ups on this method were published (see, e.g., [5, 7, 8, 18), and results were summarized in the book [6]. In this paper, we demonstrate the performance of that method for the case of experimentally measured time-dependent backscattering data for targets buried in a box filled with dry sand. This mimics the case when the targets are buried under the ground. Our primary application is in the standoff imaging of explosives, such as, e.g., land mines and improvised explosive devices (IEDs). A systematic study 
of twenty five cases conducted here shows how that method works in estimating the dielectric constants (equivalently, refractive indices) and locations of the targets. Since the technique of [6] works with the case when the targets are illuminated by a single point source, we use here a single location of the source.

This publication is a continuation of three recent works of our group, where we have treated similar experimental data for targets placed in air [10, 11, 30]. Compared with the case of targets in air, there are three main difficulties in imaging of buried targets: (i) the targets' signals are much weaker than those when the targets are in air, (ii) the targets' signals may overlap with the reflection from the ground's surface, which makes it difficult to be distinguished, and (iii) in particular for the globally convergent method, in which the Laplace transform of the timedependent data is used, the reflection from the ground's surface may dominate the targets' signals after the Laplace transform, since the kernel of the Laplace transform decays exponentially with respect to time.

The problem of subsurface imaging can be found in a variety of practical and engineering applications such as nondestructive testing, landmine and unexploded ordnance detection, archaeology, remote sensing and medical imaging, see, e.g., 23]. For migration-type methods for estimating geometric information such as shapes, sizes, and locations of targets, we refer to [28, 33. Our main goal in this work is to estimate their dielectric constants, which characterize the targets in terms of their materials. This problem is much more difficult than estimating the geometric properties. Mathematically speaking, this is a CIP for the time-dependent wave-like equation in 3-d: we reconstruct a spatially varying coefficient of that hyperbolic PDE using measurements on the backscattering part of the boundary of the domain of interest.

The most common method for solving a CIP is the least-squares approach, in which an objective functional is to be minimized using optimization methods, see, e.g., [1, 13, 14] and references therein.However, it is well known that these objective functionals are non convex and, as a rule, have multiple local minima and ravines. Therefore, the convergence of these methods cannot be rigorously guaranteed unless a good first guess of the exact solution of the CIP is chosen. This means that they require a priori knowledge of a small neighborhood of the exact solution, which is not available in many practical situations. We call these locally convergent methods. It was shown in section 5.8.4 of [6] and in [18] for transmitted timedependent experimental data with a single source that a locally convergent method starting from the homogeneous background as the first guess failed, whereas the technique of [6] worked well. A similar conclusion was independently drawn in [22] when working with a different type of experimental data.

Unlike these, it was rigorously established that the method of [6] can provide a good approximation of the exact coefficient without any a priori knowledge of a small neighborhood of this coefficient. We call a numerical method for a CIP with this property globally convergent.

Since it is extremely hard to develop globally convergent numerical methods for CIPs, the technique of [6] uses a reasonable approximate mathematical model, see section 2.3. We emphasize that this model is used only on the first iteration of that method. Due to this model, we call the method of [6] approximately globally convergent, or globally convergent, in short, see Theorem 2.9.4 in [6] and Theorem 5.1 in [7] for proofs of the global convergence in the case when a single location of the point source is used. We briefly prove here an analog of these theorems for the case when the point source is replaced by a single incident plane wave.

Apart from the three difficulties mentioned in the beginning, as we have pointed out in [30], the main challenge working with our experimental data, even when the targets are in air, is 
a huge misfit between these data and computationally simulated ones. Hence, any inversion algorithm would fail to produce satisfactory results if being applied to the raw data. Therefore, the central procedure before applying the globally convergent algorithm to the experimental data is a data preprocessing procedure, which is inevitably a heuristic one. This procedure makes the experimental data look somewhat similar to the data provided by computational simulations. In our opinion, the ultimate justification of this procedure is the accuracy of reconstruction results, especially those for blind data cases, i.e., for such sets of data for which correct answers were unknown in advance to the computational team, see section 5.1 for some details. The preprocessed data are used as the input for our globally convergent algorithm. A data preprocessing procedure, which consists of several steps, was proposed in [30. In this paper the procedure of [30] is modified to remedy the aforementioned difficulties related to the case of buried targets. In particular, we propose a new step for removing the reflection from the sand's surface. After that, the targets can be treated as being placed in air, see section 4 .

We should mention that, unlike dielectric constants, shapes of targets are more difficult to accurately reconstruct using the globally convergent method of [6]. Only their cross-sections in the measurement plane can be rather well estimated after the data propagation. To enhance the reconstruction accuracy of shapes, these results can be used as initial guesses for locally convergent methods in order to refine the images, see, e.g., [5, 6, 8, 11]. We call the combination of these two steps a two-stage numerical method. The second step requires an additional effort and will be reported in a future work. We also mention that it was demonstrated in [5, 6, 8] for the transmitted experimental data and in [11] for the backscattering experimental data that the second step refines images quite well for targets placed in air.

The rest of the paper is organized as follows. In section 2, we state the mathematical model and briefly describe the globally convergent method. In section 3 we outline the main steps of the proof of the global convergence of the proposed algorithm. Data acquisition and data preprocessing are described in section 4. In section 5 we present inversion results of the proposed globally convergent algorithm for our experimental data. Finally, some conclusions are drawn in section 6 ,

\section{Problem statement and the globally convergent method in brief}

The theory of the globally convergent method of [4] using a single point source has been thoroughly discussed in [6, 7]. The globally convergent method using a plane incident wave was stated in [30]. We briefly summarize the main ideas of the latter case here and outline in section 3 its convergence analysis, which was not included in [30].

\subsection{Problem statement}

Consider the propagation of the electromagnetic wave in $\mathbb{R}^{3}$ generated by an incident plane wave. Below, $\mathbf{x}=(x, y, z)$ denotes a point in $\mathbb{R}^{3}$. Since in our experiment only one component $E_{2}(\mathbf{x}, t)$ of the electric field $E=\left(E_{1}, E_{2}, E_{3}\right)(\mathbf{x}, t)$ is generated by the source and the detector measures only that component of the scattered electric field, we model the wave propagation by 
the following Cauchy problem for the scalar wave equation:

$$
\begin{aligned}
& \epsilon(\mathbf{x}) u_{t t}(\mathbf{x}, t)=\Delta u(\mathbf{x}, t)+\delta\left(z-z_{0}\right) f(t),(\mathbf{x}, t) \in \mathbb{R}^{3} \times(0, \infty), \\
& u(\mathbf{x}, 0)=0, \quad u_{t}(\mathbf{x}, 0)=0,
\end{aligned}
$$

where $u:=E_{2}$ is the wave generated by the incident plane wave propagating along the $z$-axis and incident at the plane $\left\{z=z_{0}\right\}$. Here $f(t) \not \equiv 0$ is a piecewise continuous bounded function representing the time-dependent waveform of the incident plane wave. It was demonstrated numerically in [3] that the component $E_{2}$ dominates components $E_{1}, E_{3}$ and that the propagation of $E_{2}$ is governed well by the solution of the Cauchy problem (11)-(2).

The coefficient $\epsilon(\mathbf{x})$ in (1) represents the spatially distributed dielectric constant of the medium in which the wave propagates. Let $\Omega \subset \mathbb{R}^{3}$ be a bounded domain and let the plane where the incident wave is emitted be located outside of the domain $\bar{\Omega}$, i.e., $\bar{\Omega} \cap\left\{z=z_{0}\right\}=\varnothing$. We assume that there exist two positive constants $0<\epsilon_{l} \leq 1$ and $\epsilon_{u} \geq 1$ such that

$$
\epsilon_{l} \leq \epsilon(\mathbf{x}) \leq \epsilon_{u}, \forall \mathbf{x} \in \mathbb{R}^{3}, \quad \epsilon(\mathbf{x}) \equiv 1, \forall \mathbf{x} \notin \Omega .
$$

In other words, the medium is assumed to be homogeneous outside of $\Omega$. We note that this assumption is not true in the case of objects buried in the ground since the dielectric constant of the ground is different from that of the air. Therefore, to use our model in this case, we first preprocess the experimental data, and then treat the buried objects as they are in air.

Below we denote by $C^{k+\alpha}$ the Hölder spaces, where $k \geq 0$ is an integer and $\alpha \in(0,1)$. Let $d>2 \epsilon_{u}$ be a constant. In addition to (3), in our theoretical analysis we assume that the function $\epsilon(\mathbf{x})$ is unknown inside $\Omega$ and

$$
\epsilon \in C^{\alpha}\left(\mathbb{R}^{3}\right), \quad\|\epsilon\|_{C^{\alpha}\left(\mathbb{R}^{3}\right)}<d,
$$

where $d>1$ is a given constant. In this work, we consider the following CIP:

CIP: Reconstruct the coefficient $\epsilon(\mathbf{x})$ for $\mathbf{x} \in \Omega$, given the following measured data for a single incident plane wave generated at the plane $\left\{z=z_{0}\right\}$ outside of $\bar{\Omega}$,

$$
g(\mathbf{x}, t)=u(\mathbf{x}, t), \mathbf{x} \in \partial \Omega, t \in(0, \infty) .
$$

For the theoretical analysis, we state the inverse problem for the case when the data are given at the entire boundary and the boundary $\partial \Omega$ being $C^{3}$-regular. However, only the backscattering data are measured in our experiment and we use a rectangular prism as $\Omega$ in our computations. As in our previous works, we complete the missing data by the solution of the forward model (11)-(2) in a homogeneous medium, see section 5 .

The assumption of the infinite time interval in (5) is not restrictive, because in our method we apply the Laplace transform to $g(\mathbf{x}, t)$ with respect to the time variable $t$. Since the kernel of this transform decays exponentially with respect to $t$, the Laplace transform effectively cuts off to zero values of the function $g(\mathbf{x}, t)$ for large $t$. Moreover, since the incident wave is excited for a finite time interval, our experimental observation has always been that the total wave almost vanishes after a finite time interval, too.

Concerning the uniqueness of this CIP, global uniqueness theorems for multidimensional CIPs with a single measurement are currently known only under the assumption that at least one initial condition does not equal zero in the entire domain $\bar{\Omega}$. Proofs of such theorems are based on Carleman estimates, see [12, 19] and sections 1.10, 1.11 in [6]. Since both initial 
conditions (22) equal zero in $\bar{\Omega}$, this method is inapplicable to our case. However, since we need to solve numerically our CIP anyway, we assume that the uniqueness holds.

We remark that (10) is invalid if metallic objects are present in the domain $\Omega$. To deal with this type of targets, we follow a suggestion of [20]. It was established numerically in [20] that metals can be modeled as dielectrics with a high dielectric constant, which is referred to as the effective dielectric constant of metals.

\subsection{The globally convergent method in brief}

The globally convergent method of [6] works with the Laplace transformed data. However, we do not invert the Laplace transform. Let

$$
\tilde{u}(\mathbf{x}, s):=(\mathcal{L} u)(\mathbf{x}, s)=\int_{0}^{\infty} u(\mathbf{x}, t) e^{-s t} d t, s \geq \underline{s}=\underline{s}(d)>0,
$$

and $\tilde{f}(s)=(\mathcal{L} f)(s)$ be the Laplace transform of $u(x, t)$ and $f(t)$, respectively, where $s$ is referred to as the pseudo frequency. We assume that $s \geq \underline{s}(d)>0$, where the number $\underline{s}(d)$ is large enough so that the Laplace transforms of the function $u$ and its derivatives $D^{\beta} u,|\beta|=1,2$, converge absolutely. The number $d$ is defined in (4). We assume that $\tilde{f}(s) \neq 0$ for all $s \geq \underline{s}(d)$. Define $w(\mathbf{x}, s):=\tilde{u}(\mathbf{x}, s) / \tilde{f}(s)$. Then, this function satisfies the equation:

$$
\Delta w(\mathbf{x}, s)-s^{2} \epsilon(\mathbf{x}) w(\mathbf{x}, s)=-\delta\left(z-z_{0}\right), \mathbf{x} \in \mathbb{R}^{3}, s \geq \underline{s}(d) .
$$

Define

$$
w_{0}(z, s)=\frac{\exp \left(-s\left|z-z_{0}\right|\right)}{2 s} .
$$

The function $w_{0}(z, s)$ is the unique solution equation (11) for the case $\epsilon(\mathbf{x}) \equiv 1$, which tends to zero as $|z| \rightarrow \infty$. It is shown in Theorem 3.1 (section 3) that in the case $f(t)=\delta(t)$

$$
\lim _{|\mathbf{x}| \rightarrow \infty}\left[w(\mathbf{x}, s)-w_{0}(z, s)\right]=0
$$

and that the function $w(\mathbf{x}, s)$ can be represented in the form

$$
w(\mathbf{x}, s)=w_{0}(z, s)+\widehat{w}(\mathbf{x}, s), \text { where } \widehat{w}(\mathbf{x}, s) \in C^{2+\alpha}\left(\mathbb{R}^{3}\right), \forall s \geq \underline{s}(d) .
$$

Furthermore, the same theorem claims that $w(\mathbf{x}, s)>0$. Thus, we assume these properties in our algorithm even if $f(t) \neq \delta(t)$. Next, define the function $v$ by $v:=(\ln w) / s^{2}$. Substituting $w=e^{v s^{2}}$ into (7) and keeping in mind that $\bar{\Omega} \cap\left\{z=z_{0}\right\}=\varnothing$, we obtain

$$
\Delta v+s^{2}|\nabla v|^{2}=\epsilon(\mathbf{x}), \mathbf{x} \in \Omega .
$$

Equation (11) shows that the coefficient $\epsilon(\mathbf{x})$ can be computed directly via the function $v$. We now eliminate the unknown coefficient $\epsilon(x)$ from equation (11) via the differentiation with respect to $s$, which is similar to the first step of the method of [12, 19]. Define the function $q$ by $q:=\partial v / \partial s$. Then $v=-\int_{s}^{\infty} q d \tau$. It follows from (11) that $q$ satisfies the following integral differential equation:

$$
\Delta q-2 s^{2} \nabla q \cdot \int_{s}^{\infty} \nabla q(\mathbf{x}, \tau) d \tau+2 s\left|\int_{s}^{\infty} \nabla q(\mathbf{x}, \tau) d \tau\right|^{2}=0, \mathbf{x} \in \Omega .
$$


Moreover, it follows from (5) that $q$ satisfies the following boundary condition:

$$
q(\mathbf{x}, s)=\psi(\mathbf{x}, s), \mathbf{x} \in \partial \Omega,
$$

where $\psi$ is derived from the boundary measured data by $\psi(\mathbf{x}, s)=\frac{\partial}{\partial s}\left[\frac{\ln (\varphi)}{s^{2}}\right]$ with $\varphi(\mathbf{x}, s)=$ $\int_{0}^{\infty} g(\mathbf{x}, t) e^{-s t} d t / \tilde{f}(s)$. To solve the problem (12), (13) for $q$, we use the following approach. We represent the integral over the infinite interval as

$$
v=-\int_{s}^{\infty} q d \tau=-\int_{s}^{\bar{s}} q d \tau+V,
$$

where $\bar{s}>\underline{s}$, which plays the role of a regularization parameter and is chosen numerically in the computational practice. The function $V(\mathbf{x}):=v(\mathbf{x}, \bar{s})$ is called the "tail function." Note that

$$
V(\mathbf{x})=\frac{\ln w(\mathbf{x}, \bar{s})}{\bar{s}^{2}} .
$$

From (12) and (14) we obtain the following nonlinear integral differential equation involving $q$ and $V$ :

$$
\begin{aligned}
\Delta q & -2 s^{2} \nabla q \cdot \int_{s}^{\bar{s}} \nabla q(\mathbf{x}, \tau) d \tau+2 s^{2} \nabla V \cdot \nabla q+2 s\left|\int_{s}^{\bar{s}} \nabla q(\mathbf{x}, \tau) d \tau\right|^{2} \\
& -4 s \nabla V \cdot \int_{s}^{\bar{s}} \nabla q(\mathbf{x}, \tau) d \tau+2 s|\nabla V|^{2}=0, \mathbf{x} \in \Omega .
\end{aligned}
$$

Note that (16) has two unknown functions $q$ and $V$. In order to approximate both of them, we use a predictor-corrector-type approach: starting from an initial guess of the tail function $V$, we solve the problem (16), (13) for $q$. After that, we calculate the coefficient $\epsilon$ via (11) and solve the forward problem for $u$. Next, we update the tail function $V$ via (15) and repeat the iterative procedure again. Thus, $V$ is the 'predictor' and $q$ is the 'corrector' here.

To approximate the integrals in (16), we make use of a layer stripping procedure with respect to $s$ described as follows. Divide the pseudo frequency interval $[\underline{s}, \bar{s}]$ into $N$ uniform subintervals by $\bar{s}=s_{0}>s_{1}>\cdots>s_{N}=\underline{s}, s_{n}-s_{n+1}=h$. We approximate $q$ by a piece-wise constant function: $q(\mathbf{x}, s) \approx q_{n}(\mathbf{x}), s \in\left(s_{n}, s_{n-1}\right], n=1, \ldots, N$. We also set $q_{0} \equiv 0$. Then after some manipulations, a system of elliptic equations for functions $q_{n}(x)$ is derived from (16) using the so-called "Carleman Weight Function" $\exp \left[\lambda\left(s-s_{n-1}\right)\right], s \in\left(s_{n}, s_{n-1}\right)$, where $\lambda \gg 1$ is a certain parameter. This system is [30]

$$
\begin{aligned}
\Delta q_{n} & +A_{1, n} \nabla q_{n} \cdot\left(\nabla V_{n}-\nabla \overline{q_{n-1}}\right) \\
& =A_{2, n}\left|\nabla q_{n}\right|^{2}+A_{3, n}\left(\left|\nabla \overline{q_{n-1}}\right|^{2}+\left|\nabla V_{n}\right|^{2}-2 \nabla V_{n} \cdot \nabla \overline{q_{n-1}}\right),
\end{aligned}
$$

where $A_{i, n}, i=1,2,3$, are some coefficients, depending on $s_{n}$ and $\lambda$, which can be analytically computed, and $\nabla \overline{q_{n-1}}=h \sum_{j=0}^{n-1} \nabla q_{j}$. Here we indicate the dependence of the tail function 
$V:=V_{n}$ on the number $n$, because we approximate $V$ iteratively. The discretized version of the boundary condition (13) is given by

$$
q_{n}(\mathbf{x})=\psi_{n}(\mathbf{x}):=\frac{1}{h} \int_{s_{n}}^{s_{n-1}} \psi(\mathbf{x}, s) d s \approx \frac{1}{2}\left[\psi\left(\mathbf{x}, s_{n}\right)+\psi\left(\mathbf{x}, s_{n-1}\right)\right], \mathbf{x} \in \partial \Omega .
$$

One can prove that $\left|A_{2, n}\right| \leq C / \lambda$ for sufficiently large $\lambda$, where $C>0$ is a certain constant. Hence, the first term in the right-hand side of (17) is dominated by the other terms. Therefore, in the following we set $A_{2, n}\left|\nabla q_{n}\right|^{2}:=0$. The system of elliptic equations (17) with boundary conditions (18) can be solved sequentially starting from $n=1$. To solve it, we make use of the iterative process: For a given $n$ and some approximation $q_{n, i-1}$ of $q_{n}$, we find the next approximation $q_{n, i}$ of $q_{n}$ by solving the Dirichlet boundary value problem (17)-(18) with $q_{n}$ on the right-hand side replaced by $q_{n, i-1}$. Denote by $m_{n}$ the number of these iterations.

Let $\Omega^{\prime} \subset \Omega$ be a certain subdomain with $\partial \Omega^{\prime} \cap \partial \Omega=\varnothing$. This subdomain is chosen computationally. Choose a function $\chi(\mathbf{x}) \in C^{1}\left(\mathbb{R}^{3}\right)$ such that

$$
\chi(\mathbf{x})=\left\{\begin{array}{c}
1, \mathbf{x} \in \Omega^{\prime} \\
\in[0,1], \mathbf{x} \in \Omega \backslash \Omega^{\prime}, \\
0, \mathbf{x} \in \mathbb{R}^{3} \backslash \Omega .
\end{array}\right.
$$

The steps of the globally convergent algorithm is summarized as follows.

- Given the first tail $V_{1,1}:=V_{0}$. Set $q_{0} \equiv 0$.

- For $n=1,2, \ldots, N$

1. Set $q_{n, 0}=q_{n-1}, V_{n, 1}=V_{n-1}$.

2. For $i=1,2, \ldots, m_{n}$

- Find $q_{n, i}$ by solving the problem (17), (18) with $V_{n}:=V_{n, i}$.

- Compute $v_{n, i}=-h q_{n, i}-\overline{q_{n-1}}+V_{n, i}, \mathbf{x} \in \Omega$.

- Compute $\epsilon_{n, i}$ via (11). Then set $\bar{\epsilon}_{n, i}(\mathbf{x})=(1-\chi(\mathbf{x}))+\chi(\mathbf{x}) \epsilon_{n, i}(\mathbf{x})$. Next, solve the forward problem (11), (2) with the new computed coefficient $\epsilon:=\bar{\epsilon}_{n, i}$, compute $w:=w_{n, i}$ and update the tail $V_{n, i+1}$ via (15).

3. Set $q_{n}=q_{n, m_{n}}, \epsilon_{n}=\epsilon_{n, m_{n}}, V_{n}=V_{n, m_{n}+1}$ and go to the next frequency interval $\left[s_{n+1}, s_{n}\right]$ if $n<N$. If one of the stopping criteria is satisfied at $n:=\bar{N} \in[1, N]$ or $n=N$, then stop.

In computations, the number of inner iterations $m_{n}$ is determined by the stopping criterion with respect to $i$. In this paper, we use the stopping criteria with respect to $i, n$ proposed in [10, 30], see a brief description in Section 5.

\subsection{The initial tail function}

We remark that the convergence of this algorithm depends on the choice of the initial tail function $V_{1,1}$. To ensure the global convergence, the choice of this function should not rely on any a priori knowledge of the exact solution of our CIP. First, in accordance with the Tikhonov 
approach to ill-posed problems (see, e.g., section 1.4 in [6]), we assume the existence of the exact solution $\epsilon^{*}(\mathbf{x})$ of our CIP for noiseless data $g^{*}(\mathbf{x}, t)$ in (5). We assume that the function $\epsilon^{*}(\mathbf{x})$ satisfies conditions (3), (4). Let $V^{*}(\mathbf{x}, s)$ and $w^{*}(\mathbf{x}, s)$ be respectively the tail function and the function $w$ which correspond to $\epsilon^{*}(\mathbf{x})$. In [25] the solution of a general hyperbolic equation is constructed for the case when the wave is generated by the plane wave. Using results of [25], one can prove, that under some conditions, there exists a function $p^{*}(\mathbf{x}) \in C^{2+\alpha}(\bar{\Omega})$ such that $V^{*}(\mathbf{x}, s)=p^{*}(\mathbf{x}) / s+O\left(1 / s^{2}\right), s \rightarrow \infty$. The proof is similar to the proof of an analogous formula (5.3.17) in [19], which has used the construction of [24] of the solution of a general hyperbolic equation for the case of the point source.

Due to this asymptotic behavior, we introduce our approximate mathematical model via the assumption that the exact tail is given by

$$
V^{*}(\mathbf{x}, s)=\frac{p^{*}(\mathbf{x})}{s}=\frac{\ln w^{*}(\mathbf{x}, s)}{s^{2}}, \forall s \geq \bar{s} .
$$

Since $q(\mathbf{x}, s)=\partial_{s} V(\mathbf{x}, s)$, then (19) implies that $q^{*}(\mathbf{x}, s)=-p^{*}(\mathbf{x}) / s^{2}, \forall s \geq \bar{s}$. Note that we use this assumption only on the initializing iteration to obtain $V_{1,1}(\mathbf{x})$. Substituting these expressions for $V^{*}, q^{*}$ at $s=\bar{s}$ in (13), (16), we obtain that the function $p^{*}(\mathbf{x})$ satisfies

$$
\begin{aligned}
\Delta p^{*}(\mathbf{x}) & =0, \mathbf{x} \in \Omega, \quad p^{*} \in C^{2+\alpha}(\bar{\Omega}), \\
\left.p^{*}\right|_{\partial \Omega} & =-\bar{s}^{2} \psi^{*}(\mathbf{x}, \bar{s}),
\end{aligned}
$$

where $\psi^{*}(\mathbf{x}, s)$ is the function in (13) for the case of the exact data. Since actually we have the function $\psi(\mathbf{x}, s)$, which is supposed to be contaminated by noise, then as the first guess for the tail function we take

$$
V_{1,1}(\mathbf{x}):=\frac{p(\mathbf{x})}{\bar{s}}, \mathbf{x} \in \Omega,
$$

where the function $p(\mathbf{x})$ is the solution of the problem (20), (21) for the case when $\psi^{*}(\mathbf{x}, \bar{s})$ is replaced by $\psi(\mathbf{x}, \bar{s})$,

$$
\begin{aligned}
\Delta p(\mathbf{x}) & =0, \mathbf{x} \in \Omega, \\
\left.p\right|_{\partial \Omega} & =-\bar{s}^{2} \psi(\mathbf{x}, \bar{s}) .
\end{aligned}
$$

\section{Global convergence}

In this section we briefly discuss the question of the approximate global convergence of the above numerical method. The only difference between this convergence analysis and the one in Theorem 2.9.4 in [6] and Theorem 5.1 in [7] is that we now use the plane wave instead of the point source. This causes the difference only in the proof of Theorem 2.7.2 of [6]. Theorem 3.1] is a direct analog of Theorem 2.7.2 of [6] for the case of the plane wave. For brevity we point here only to those features of the proof of Theorem 3.1 which are different from those of Theorem 2.7.2 of [6]. Next, for the convenience of the reader, we briefly outline the proof of Theorem [3.2. since it is completely the same as proofs of Theorem 5.1 in [7] and Theorem 2.9.4 in [6]. To follow more closely arguments of [6, 7], we assume in our convergence analysis that the lower bound $\epsilon_{l}$ in (3) is chosen by

$$
\epsilon_{l}=1
$$


Theorem 3.1. Let the function $\epsilon(\mathbf{x})$ satisfies conditions (3), (4) and (25) and let in (11) $f(t)=$ $\delta(t)$. Then there exists a number $\underline{s}(d)>0$ such that for every $s>\underline{s}(d)$ the function $w(\mathbf{x}, s)=$ $\mathcal{L}(u)(\mathbf{x}, s)$ satisfies conditions (7)-(10), where the operator $\mathcal{L}$ is defined in (6)). Furthermore,

$$
w_{u}(z, s)<w(\mathbf{x}, s) \leq w_{0}(z, s),
$$

where the function $w_{0}(z, s)$ is defined in (8) and $w_{u}(z, s)$ is such a solution of (7) for $\epsilon(\mathbf{x}) \equiv \epsilon_{u}$ which tends to zero as $|z| \rightarrow \infty$, i.e.,

$$
w_{u}(z, s)=\exp \left(-s \sqrt{\epsilon_{u}}\left|z-z_{0}\right|\right) /\left(2 s \sqrt{\epsilon_{u}}\right) .
$$

Also, the solution of the problem (7)-(10) is unique.

Proof. Note that the classical theory of elliptic equations [17] is not directly applicable here, since it works only with bounded domains, whereas we work here with (17) in the entire space $\mathbb{R}^{3}$. This causes additional difficulties of the proof. In this proof, we consider $s>\underline{s}(d)$.

It is well known that in the case $f(t)=\delta(t)$ the Cauchy problem (11), (2) is equivalent to the Cauchy problem for the homogeneous equation (11) with initial conditions $u(x, 0)=0, u_{t}(x, 0)=$ $\delta\left(z-z_{0}\right)$ [31. Consider the well known Laplace-like transform, which transforms the Cauchy problem for the hyperbolic equation in the Cauchy problem for a similar parabolic equation (see, e.g., formula (1.162) in [6]),

$$
v(\mathbf{x}, t):=\left(\mathcal{L}_{1} u\right)(\mathbf{x}, t):=\frac{1}{2 \sqrt{\pi} t^{3 / 2}} \int_{0}^{\infty} u(\mathbf{x}, \tau) \tau \exp \left(-\frac{\tau^{2}}{4 t}\right) d \tau, t>0 .
$$

Then $v(\mathbf{x}, t)$ is the solution of the following parabolic Cauchy problem

$$
\begin{aligned}
& \epsilon(\mathbf{x}) v_{t}=\Delta v, \quad(\mathbf{x}, t) \in \mathbb{R}^{3} \times(0, \infty) \\
& v(\mathbf{x}, 0)=\delta\left(z-z_{0}\right) .
\end{aligned}
$$

Also, define another analog of the Laplace transform,

$$
\left(\mathcal{L}_{2} v\right)(\mathbf{x}, s)=\int_{0}^{\infty} v(\mathbf{x}, t) e^{-s^{2} t} d t, s>0
$$

By the formula (28) of section 4.5 of tables of the Laplace transform [2]

$$
\int_{0}^{\infty} e^{-s^{2} t}\left[\frac{1}{2 \sqrt{\pi} t^{3 / 2}} \tau \exp \left(-\frac{\tau^{2}}{4 t}\right)\right] d t=e^{-s \tau} ; s, \tau>0 .
$$

Hence, using (6) and (29), we obtain for sufficiently large $s>0$

$$
w(\mathbf{x}, s)=(\mathcal{L} u)(\mathbf{x}, s)=\mathcal{L}_{2}\left(\mathcal{L}_{1} u\right)(\mathbf{x}, s)=\left(\mathcal{L}_{2} v\right)(\mathbf{x}, s) .
$$

Hence, we study now the problem (27), (28). Let $Z(\mathbf{x}, \xi, t, \tau)$ be the fundamental solution for the parabolic operator $\epsilon(\mathbf{x}) \partial_{t}-\Delta$. Then the formula (13.1) of Chapter 4 of [21] along with other 
detailed estimates of the fundamental solution of the general second order parabolic equation given in $\S 11-\S 14$ of Chapter 4 of [21] imply that for $2 r+|\gamma| \leq 2, t>\tau$

$$
\left|D_{t}^{r} D_{\mathbf{x}}^{\gamma} Z(\mathbf{x}, \xi, t, \tau)\right| \leq \frac{\exp (M t)}{(t-\tau)^{(3+2 r+|\gamma|) / 2}} \exp \left(-C \frac{|\mathbf{x}-\xi|^{2}}{t-\tau}\right) .
$$

Here and below $M=M(d)>0$ and $C=C(d)>0$ denote different numbers depending only on the number $d$. By (27), (28)

$$
v(\mathbf{x}, t)=\int_{\mathbb{R}^{3}} Z(\mathbf{x}, \xi, t, 0) \delta\left(\xi_{3}-z_{0}\right) d \xi=\int_{\mathbb{R}^{2}} Z\left(\mathbf{x}, \xi_{1}, \xi_{2}, z_{0}, t, 0\right) d \xi_{1} d \xi_{2} .
$$

Hence, (31) implies that

$$
\left|D_{t}^{r} D_{\mathbf{x}}^{\gamma} v(\mathbf{x}, t)\right| \leq \frac{\exp (M t)}{t^{(1+2 r+|\gamma|) / 2}} \exp \left(-C \frac{\left(z-z_{0}\right)^{2}}{t}\right) .
$$

Hence, one can apply the operator $\mathcal{L}_{2}$ to the functions $D_{t}^{r} D_{\mathbf{x}}^{\gamma} v(\mathbf{x}, t)$ for $s \geq \underline{s}(d)=\sqrt{M(d)}$. Hence, (27), (28) and (30) imply that the function $w$ satisfies (7).

We now prove that the function $w$ satisfies conditions (9), (10). Let

$$
v_{0}(z, t)=\exp \left[-\left(z-z_{0}\right)^{2} /(4 t)\right] /(2 \sqrt{\pi t})
$$

be the solution of the problem (27), (28) for the case $\epsilon(\mathbf{x}) \equiv 1$. Denote $\widetilde{v}(\mathbf{x}, t)=v(\mathbf{x}, t)-v_{0}(z, t)$. Then

$$
\epsilon(\mathbf{x}) \widetilde{v}_{t}-\Delta \widetilde{v}=[1-\epsilon(\mathbf{x})] v_{0 t}, \quad \widetilde{v}(\mathbf{x}, 0)=0 .
$$

Since $\epsilon(\mathbf{x})=1$ for $\mathbf{x} \notin \Omega$ and $\Omega \cap\left\{z=z_{0}\right\}=\varnothing$, then the right-hand side of equation (32) does not have a singularity. Hence, (32) implies that

$$
\widetilde{v}(\mathbf{x}, t)=\int_{0}^{t} d \tau \int_{\Omega} Z(\mathbf{x}, \xi, t, \tau)[1-\epsilon(\xi)] v_{0 \tau}\left(\xi_{3}, \tau\right) d \xi .
$$

Hence, it follows from (31) that

$$
|\widetilde{v}(\mathbf{x}, t)| \leq \exp (M t) \int_{0}^{t} d \tau \int_{\Omega} \frac{1}{(t-\tau)^{3 / 2}} \exp \left(-C \frac{|\mathbf{x}-\xi|^{2}}{t-\tau}\right)\left|v_{0 \tau}\left(\xi_{3}, \tau\right)\right| d \xi .
$$

Also,

$$
\left|v_{0 \tau}\left(\xi_{3}, \tau\right)\right| \leq C_{1}\left[\frac{\left(\xi_{3}-z_{0}\right)^{2}}{\tau^{5 / 2}}+\frac{1}{\tau^{3 / 2}}\right] \exp \left[-\frac{\left(\xi_{3}-z_{0}\right)^{2}}{4 \tau}\right],
$$

where the number $C_{1}>0$ is independent of $\xi_{3}, z_{0}, \tau$. Using formulas (28) and (29) of section 4.5 of [2], we obtain

$$
\mathcal{L}_{2}\left[\frac{1}{t^{3 / 2}} \exp \left(-C \frac{|\mathbf{x}-\xi|^{2}}{t}\right)\right]=\frac{\sqrt{\pi} \exp [-2 \sqrt{C} s|\mathbf{x}-\xi|]}{\sqrt{C}|\mathbf{x}-\xi|}
$$




$$
\begin{gathered}
\mathcal{L}_{2}\left\{\frac{1}{t^{3 / 2}} \exp \left[-\frac{\left(\xi_{3}-z_{0}\right)^{2}}{4 t}\right]\right\}=\frac{2 \sqrt{\pi}}{\left|\xi_{3}-z_{0}\right|} \exp \left(-s\left|\xi_{3}-z_{0}\right|\right), \\
\mathcal{L}_{2}\left\{\frac{\left(\xi_{3}-z_{0}\right)^{2}}{t^{5 / 2}} \exp \left[-\frac{\left(\xi_{3}-z_{0}\right)^{2}}{4 t}\right]\right\}=\frac{16 s^{3}}{\left|\xi_{3}-z_{0}\right|} K_{3 / 2}\left(s\left|\xi_{3}-z_{0}\right|\right),
\end{gathered}
$$

where $K_{3 / 2}$ is the McDonald function. Since $\bar{\Omega} \cap\left\{z=z_{0}\right\}=\varnothing$, then the right-hand sides of formulas (36) and (37) do not have a singularity for $\xi \in \Omega$.

By the formula $(27)$ of section 4.5 of $[2]\left(\mathcal{L}_{2} v_{0}\right)(z, s)=w_{0}(z, s)$. Hence, let $\widehat{w}(\mathbf{x}, s)=$ $w(\mathbf{x}, s)-w_{0}(z, s)=\left(\mathcal{L}_{2} \widetilde{v}\right)(\mathbf{x}, s)$. Since $\left|\left(\mathcal{L}_{2} \widetilde{v}\right)(\mathbf{x}, s)\right| \leq\left(\mathcal{L}_{2}(|\widetilde{v}|)\right)(\mathbf{x}, s)$, then (33)-(37) and the convolution theorem for the Laplace transform imply that

$$
\begin{aligned}
|\widehat{w}(\mathbf{x}, s)| & \leq C_{2} s^{3} \int_{\Omega} \frac{\exp (-2 \sqrt{C} s|\mathbf{x}-\xi|)}{|\mathbf{x}-\xi|}\left[\frac{\exp \left(-s\left|\xi_{3}-z_{0}\right|\right)}{\left|\xi_{3}-z_{0}\right|}+\frac{K_{3 / 2}\left(s\left|\xi_{3}-z_{0}\right|\right)}{\left|\xi_{3}-z_{0}\right|}\right] d \xi \\
& \leq C_{2} \exp \left(-C_{3}(s-\sqrt{M(d)})|\mathbf{x}|\right), s>\underline{s}(d)=\sqrt{M(d)},|\mathbf{x}| \rightarrow \infty,
\end{aligned}
$$

where numbers $C_{2}=C_{2}\left(C, \Omega, z_{0}\right)>0, C_{3}=C_{3}\left(C, \Omega, z_{0}\right)>0$ depend only on the listed parameters. Therefore, condition (9) holds.

Next, the function $\widehat{w}(\mathbf{x}, s)$ satisfies the following conditions:

$$
\begin{aligned}
\Delta \widehat{w}-s^{2} \epsilon(\mathbf{x}) \widehat{w} & =s^{2}(\epsilon(\mathbf{x})-1) w_{0}(z, s), s>\underline{s}(d)=\sqrt{M(d)}, \\
\lim _{|x| \rightarrow \infty} \widehat{w}(\mathbf{x}, s) & =0 .
\end{aligned}
$$

Since the right-hand side of (38) belongs to $C^{\alpha}\left(\mathbb{R}^{3}\right)$, then $\widehat{w}(\mathbf{x}, s) \in C^{2+\alpha}(\bar{G})$ for every bounded domain $G \subset \mathbb{R}^{3}$ [17. In particular, this implies that

$$
w(\mathbf{x}, s)=w_{0}(z, s)-s^{2} \int_{\Omega} \frac{\exp (-s|\mathbf{x}-\xi|)}{4 \pi|\mathbf{x}-\xi|}[\epsilon(\xi)-1] w(\xi, s) d \xi .
$$

It is clear from (40) that the function $\widehat{w}(\mathbf{x}, s) \in C^{\infty}\left(\mathbb{R}^{3} \backslash \widetilde{\Omega}\right)$ for any bounded domain $\widetilde{\Omega}$ such that $\Omega \subset \widetilde{\Omega}, \partial \Omega \cap \partial \widetilde{\Omega}=\varnothing$. It is also clear that this function decays exponentially together with its derivatives as $|\mathbf{x}| \rightarrow \infty$. Hence, $\widehat{w}(\mathbf{x}, s) \in C^{2+\alpha}\left(\mathbb{R}^{3}\right)$. Thus, we have established that the function $w$ satisfies conditions (91), (10).

We now prove uniqueness of the solution of the problem (7)-(10). Assume that there exist two solutions of this problem, $w_{1}$ and $w_{2}$. Then both of them can be represented via (40). Hence, $w_{1}-w_{0}:=\widehat{w}_{1} \in H^{1}\left(\mathbb{R}^{3}\right)$ and $w_{2}-w_{0}=\widehat{w}_{2} \in H^{1}\left(\mathbb{R}^{3}\right)$. Furthermore, both functions $\widehat{w}_{1}, \widehat{w}_{2}$ decay exponentially together with their derivatives as $|\mathbf{x}| \rightarrow \infty$. On the other hand, since both these functions satisfy conditions (38), (39), then subtracting equation (38) for $\widehat{w}_{2}$ from the same equation for $\widehat{w}_{1}$, multiplying the resulting equation by $\widehat{w}_{1}-\widehat{w}_{2}$, integrating over $\mathbb{R}^{3}$ and using integration by parts, we obtain in a standard manner that $\widehat{w}_{1}-\widehat{w}_{2} \equiv 0$. Thus, uniqueness is established.

We now prove estimates (26). For an arbitrary number $R>0$ let $B_{R}=\{|\mathbf{x}|<R\}$. Since the function $(\epsilon(\mathbf{x})-1) w_{0}(z, s) \geq 0$, then the maximum principle applied to (38) implies that if $\max _{\bar{B}_{R}} \widehat{w}(\mathbf{x}, s)>0$, then this maximum is achieved at a point $\mathbf{x}_{0}(R) \in \partial B_{R}$. Hence, setting $R \rightarrow \infty$ and using (39), we obtain $\widehat{w}(\mathbf{x}, s) \leq 0$, which proves the right inequality (26). 
We now prove the left inequality (26). Let

$$
v_{u}(z, t)=\frac{\sqrt{\epsilon_{u}}}{2 \sqrt{\pi t}} \exp \left[-\frac{\epsilon_{u}\left(z-z_{0}\right)^{2}}{4 t}\right]
$$

be the solution of the problem (27)-(28) for $\epsilon(\mathbf{x}) \equiv \epsilon_{u}$. Let

$$
p(\mathbf{x}, t)=\int_{0}^{t}\left(v(\mathbf{x}, \tau)-v_{u}(z, \tau)\right) d \tau .
$$

Then $\epsilon(\mathbf{x}) p_{t}-\Delta p=\left(\epsilon(\mathbf{x})-\epsilon_{u}\right) v_{u}$ and $p(\mathbf{x}, 0)=0$. Since $\left(\epsilon(\mathbf{x})-\epsilon_{u}\right) v_{u}>0$ for $t>0$, then the function $p(\mathbf{x}, t)$ is not identical zero. Hence, the maximum principle of Theorem 1 of Chapter 2 of [16] implies that $p(\mathbf{x}, t)>0$ for $t>0$. Hence, $\left(\mathcal{L}_{2} p\right)(\mathbf{x}, s)>0$. On the other hand,

$$
\left(\mathcal{L}_{2} p\right)(\mathbf{x}, s)=\frac{1}{s^{2}}\left[\left(\mathcal{L}_{2} v\right)(\mathbf{x}, s)-\left(\mathcal{L}_{2} v_{u}\right)(z, s)\right]=\frac{1}{s^{2}}\left[w(\mathbf{x}, s)-w_{u}(z, s)\right]>0 .
$$

The formulation of the global convergence Theorem 3.2 is similar to the formulation of Theorem 5.1 in [7]. First, the function $q^{*}(\mathbf{x}, s) \in C^{2+\alpha}(\bar{\Omega})$ is introduced as well as its Dirichlet boundary condition $\psi^{*}(\mathbf{x}, s)=\left.q^{*}(\mathbf{x}, s)\right|_{\partial \Omega}$, where $q^{*}(\mathbf{x}, s) \in C^{2+\alpha}(\bar{\Omega}), \psi^{*}(\mathbf{x}, s) \in C^{2+\alpha}(\partial \Omega), \forall s \in$ $[\underline{s}, \bar{s}]$. These functions correspond to the exact coefficient $\epsilon^{*}(\mathbf{x})$. We also assume that the function $\psi(\mathbf{x}, s) \in C^{2+\alpha}(\partial \Omega), \forall s \in[\underline{s}, \bar{s}]$. Next, similarly to functions $q_{n}(\mathbf{x})$ and $\psi_{n}(\mathbf{x})$, functions $q_{n}^{*}(\mathbf{x}) \in C^{2+\alpha}(\bar{\Omega})$ and $\psi_{n}^{*}(\mathbf{x}) \in C^{2+\alpha}(\partial \Omega)$ are obtained from functions $q^{*}(\mathbf{x}, s)$ and $\psi^{*}(\mathbf{x}, s)$, respectively. The functions $q_{n}^{*}$ are solutions of analogs for equations (17) with Dirichlet boundary conditions $\psi_{n}^{*}$. There exists a constant $C^{*}=C^{*}\left(\epsilon_{u}, d\right)>1$ such that

$$
\begin{aligned}
& \left\|q^{*}(\mathbf{x}, s)-q_{n}^{*}(\mathbf{x})\right\|_{C^{2+\alpha}(\bar{\Omega})}+\left\|\psi^{*}(\mathbf{x}, s)-\psi_{n}^{*}(\mathbf{x})\right\|_{C^{2+\alpha}(\partial \Omega)} \\
\leq & C^{*} h, s \in\left(s_{n}, s_{n-1}\right], n=1, \ldots, N .
\end{aligned}
$$

Let $\sigma \in(0,1)$ be the level of the error in the boundary data $\psi(\mathbf{x}, s)$,

$$
\left\|\psi(\mathbf{x}, s)-\psi^{*}(\mathbf{x}, s)\right\|_{C^{2+\alpha}(\partial \Omega)} \leq C^{*} \sigma, \forall s \in[\underline{s}, \bar{s}] .
$$

Introduce the error parameter $\eta=h+\sigma$. Then (18), (42) and (43) imply that it is natural to assume that

$$
\left\|\psi_{n}-\psi_{n}^{*}\right\|_{C^{2+\alpha}(\partial \Omega)} \leq C^{*} \eta, s \in\left(s_{n}, s_{n-1}\right], n=1, \ldots, N .
$$

Theorem 3.2 (Global convergence). Assume that in (1) $f(t)=\delta(t)$, the approximation (19) holds and that the initial tail function $V_{1,1}(\mathbf{x})$ is calculated as in section 2.3. Suppose that the maximal number of iterations with respect to $i$ is $m \geq 1$ and that the algorithm is stopped at a certain $n=\bar{N} \in[1, N]$. Also, let (25), (43) and (44) hold. In addition, assume that all functions $\epsilon_{n, i}$ satisfy $\epsilon_{n, i}(\mathbf{x}) \geq 1$ and that $\left\|\epsilon^{*}\right\|_{C^{\alpha}\left(\mathbb{R}^{3}\right)} \leq d / 2$. Also, let $\underline{s} \geq \underline{s}(d)$ and $\bar{s}>1$. Then there exists a number $B=B\left(\Omega, \chi, \bar{s}, \alpha, \epsilon_{u}, d\right)>1$ depending only on listed parameters such that if the error parameter $\eta$ is so small that

$$
\eta \in\left(0, \eta_{0}\right), \eta_{0}=\frac{1}{B^{6 N m}}
$$

then for $i \in[1, m], n \in[1, \bar{N}]$

$$
\left\|\epsilon_{n, i}-\epsilon^{*}\right\|_{C^{\alpha}(\bar{\Omega})} \leq B^{3(i+(n-1) m)} \eta \leq \sqrt{\eta} .
$$


Outline of the proof. This proof is similar to the proofs of Theorem 2.9.4 in [6] and Theorem 5.1 in [7]. Denote by $P_{n, i}=\left\|\nabla V_{n, i}-\nabla V^{*}\right\|_{C^{1+\alpha}(\bar{\Omega})}, Q_{n, i}=\left\|q_{n, i}-q_{n}^{*}\right\|_{C^{2+\alpha}(\bar{\Omega})}, R_{n, i}=$ $\left\|\epsilon_{n, i}-\epsilon^{*}\right\|_{C^{\alpha}(\bar{\Omega})}$. First, $P_{1,1}$ is estimated using (19)-(24) and Schauder theorem [17, 21]. Next, $Q_{1,1}$ is estimated using Schauder theorem and (44). Next, the number $R_{1,1}$ is estimated using estimates for $P_{1,1}$ and $Q_{1,1}$ as well as equation (11): recall that functions $\epsilon_{n, i}$ are reconstructed via (11). On each follow up iterative step $(n, i)$ we first estimate $P_{n, i}$. Next, we use Schauder theorem to estimate $Q_{n, i}$. And finally we use (111) to estimate $R_{n, i}$.

We now outline an idea of estimating numbers $P_{n, i}$. Using (15), we obtain that for $(n, i) \neq$ $(1,1)$

$$
\nabla V_{n, i}(\mathbf{x})-\nabla V^{*}(\mathbf{x})=\frac{\left[\left(\nabla w_{n, i}-\nabla w^{*}\right) w^{*}\right](\mathbf{x}, \bar{s})+\left[\left(w^{*}-w_{n, i}\right) \nabla w^{*}\right](\mathbf{x}, \bar{s})}{\left(w_{n, i} w^{*}\right)(\mathbf{x}, \bar{s})} .
$$

A similar formula is also valid for $\Delta V_{n, i}(\mathbf{x})-\Delta V^{*}(\mathbf{x})$. Hence, using the left estimate (26), we obtain that in (46) $0<1 /\left(w_{n, i} w^{*}\right)(\mathbf{x}, \bar{s}) \leq B_{1}$ with a certain number $B_{1}>0$ depending on the same parameters as the number $B$. Next, using the right estimate (26) as well as arguments of Theorem 2.9.1.2 of [6], we obtain from (46) an estimate for $P_{n, i}$ via $R_{n, i-1}$.

Remark 3.1. Theorem 3.2 states that if the total number of iterations with respect to both parameters $n, i$ does not exceed $N m$ and if the error parameter $\eta$ is sufficiently small, then all functions $\epsilon_{n, i}$ are located in a sufficiently small neighborhood of the exact solution $\epsilon^{*}$. The size of this neighborhood is defined only by the error parameter, which is natural. This property holds regardless on any a priori knowledge of that small neighborhood. Therefore we have global convergence within the framework of the approximate mathematical model of section 2.3.

From the computational standpoint, even though the error estimate 45) is valid at the first iteration, our numerical observations suggest that the algorithm should be continued after the first iteration. The reason is that (45) is valid only for the approximate mathematical model (19). As to which of functions $\epsilon_{n, i}$ one should consider as the final solution of our inverse problem, it should be decided on the basis of a stopping criterion, which is chosen computationally. It is important that we use the same stopping criterion for all our numerical tests described below. We also note that it is a well known phenomenon in the field of Ill-Posed Problems that the iteration number is chosen as one of regularization parameters, see, e.g. pages 156, 157 of [14]. The approximate mathematical model (19) should be verified computationally. This was done in above cited works of our group as well as in the current paper.

\section{Data acquisition and preprocessing}

\subsection{Data acquisition}

The collection of the experimental data was carried out using the same configuration as in [10, 30], where we imaged targets placed in air, see the detailed description in section 3 of [30]. The only difference is that in the current paper the objects considered were placed inside a box filled with dry sand with the dielectric constant of $\epsilon$ (sand) $=4$. This was used to model the case of buried objects, see Figure 1 .

The sand box was placed in front of the transmitter. Its front and back sides were covered by Styrofoam whose dielectric constant is approximately 1, i.e., it does not affect the incident and scattered signals. The transmitter (a horn antenna) was fixed at a given position while a detector was scanned over a square of a vertical plane behind the transmitter, which we refer 
to as the measurement plane. At each detector location, the source emitted an electric pulse of 300 picoseconds (ps) duration, and the time-dependent scattered wave was captured by the detector. Then the detector was moved to the next location and the measurement was repeated. Hence, our data can be considered as generated by a single point source. Consider the Cartesian coordinate system 0xyz as shown in Figure1(b). Below "m" stands for meter. The detector was scanned in the square of $1 \mathrm{~m}$ by $1 \mathrm{~m}$ with the step size of $0.02 \mathrm{~m}$, starting at $(x, y)=(-0.5,-0.5)$ and ending at $(x, y)=(0.5,0.5)$. The horn antenna was placed at the distance of about $0.2-0.25$ $\mathrm{m}$ from the measurement plane, and the distance from the sand box to the measurement plane was about $0.7-0.8 \mathrm{~m}$.

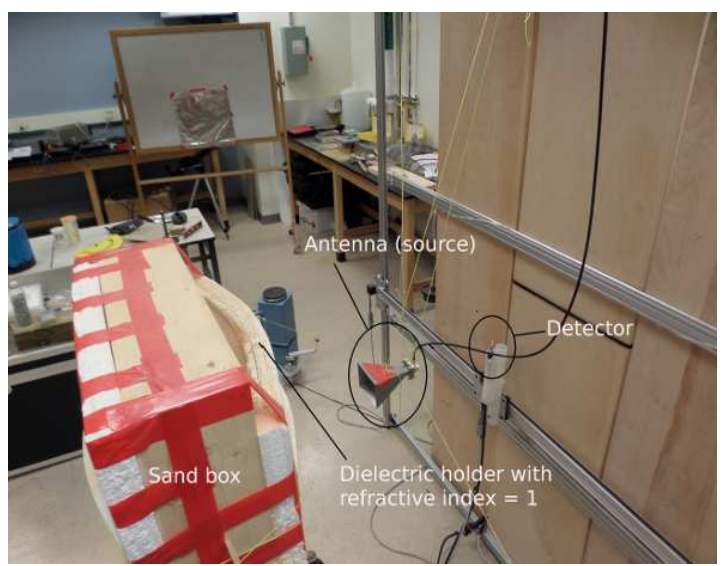

(a)

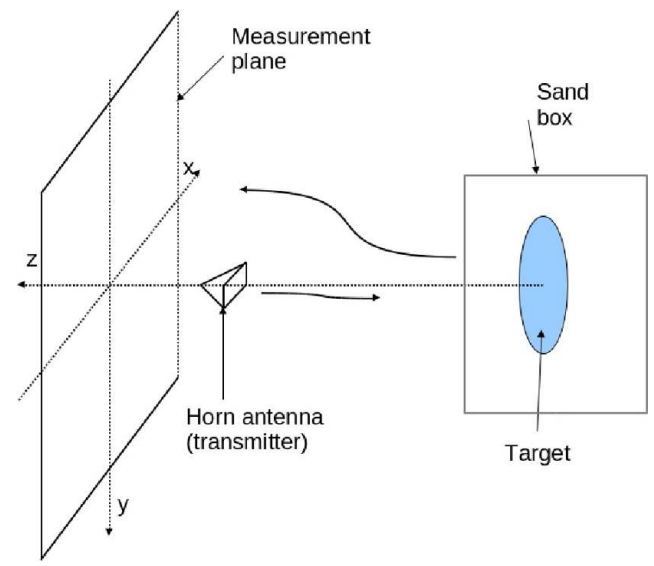

(b)

Figure 1: (a): Experimental setup; (b) Schematic diagram of our data acquisition.

The wavelength of the incident pulses was about $0.04 \mathrm{~m}$. The time step between two consecutive records was $\Delta t=10 \mathrm{ps}$. Each signal was recorded for 10 nanoseconds. Since the source was located far away (at about 12-15 wavelengths) from the targets, the use of the incident plane wave in our model (1)-(2) is well suited.

\subsection{Data preprocessing}

As we have mentioned in Introduction, there is a huge misfit between our experimental data and the data produced via computational simulations. We have pointed out in [30] that there are several causes of this misfit such as: (i) the instability of the amplitude of the emitted signals which causes the instability of the backscatter signals, (ii) unwanted waves scattered by several existing objects around our device (see Figure 3.2(a) of [30]), (iii) the shadow on the measurement plane caused by the horn antenna since it was placed between the sand box and the measurement plane, see Figures 1, and (iv) the difference between the experimental and simulated incident waves. Due to some limitations, it was technically very hard to place the horn antenna behind the measurement plane. Therefore, the central procedure required before applying inversion methods is data preprocessing. This procedure is inevitably heuristic and cannot be rigorously justified. In the case of targets located in air we proposed in [30] a data preprocessing procedure which consists of the following steps:

1. Off-set correction: The recorded signals may be shifted from the zero mean value. This can be corrected by subtracting the mean value from them. 
2. Time-zero correction: Time-zero refers to the moment at which an incident signal is emitted from the transmitter. This step shifts the data to the correct time-zero.

3. Data propagation: In this step, the data is propagated to a plane, which we refer to as the propagated plane. This plane is located closer to the targets than the measurement plane. This means that we approximate the scattered wave on the propagated plane using the measured scattered wave on the measurement plane. There are two reasons for doing this [30]. The first one is that since the kernel of the Laplace transform (6) decays exponentially with respect to time, which is proportional to the distance from the targets to the measurement plane, then the amplitude of the data after the Laplace transform on the measurement plane is very small and can be dominated by the computational error. The second reason is that the data propagation procedure helps to reduce the computational cost substantially since the size of the computational domain $\Omega$ is reduced. We have also observed that the data propagation helps to reduce the noise in the measured data.

4. Extraction of target's signal: This is the most difficult step of data preprocessing. Apart from the signals scattered from the targets, our measured data also contain various types of unwanted signals and noise. The unwanted signals which come earlier than the targets' signals dominate the latter after the Laplace transform. Thus, this step helps to remove both these unwanted signals and noise. In addition to unwanted signals and noise as in the case of targets located in air, in our situation of targets buried in the sand this step also removes the signal caused by the sand, see section 4.2.2.

5. Source shift: In our simulation, we assume that the incident plane wave is emitted on the $\left\{z=z_{0}\right\}$. Therefore, the computational cost depends on the distance from this plane to our targets. To avoid unnecessary computational cost in our forward and inverse solvers, we artificially shift the source closer to the sand box. This is done by shifting the whole timedependent data in time.

6. Data calibration: Finally, since the amplitude of the experimental signals are usually quite different from the simulated ones, we scale the former to better match the latter in amplitude by multiplying the former by a certain factor, which we call the calibration factor. The choice of this factor is based on the data for a known target referred to as the calibrating object.

Steps 1, 2, 5 and 6 are basically the same as in [30]. We just note that, in this paper, only one calibration factor was used in Step 6 for both non metallic and metallic targets in contrast to the data used in [10, 30, where two different factors were used for these two types of target. However, steps 3 and 4 used in this paper are different from those of the above works, therefore we present them below. Moreover, in Step 4 we also present a method for estimating the burial depth of a target, which is defined as the distance from the front surface of the target to the sand's surface.

\subsubsection{Data propagation}

In [30] we proposed a time-reversal data propagation method. The idea of that method is to solve a time-domain wave equation with the reversed time variable. In this paper, we make use of another data propagation method which is based on the data back-propagation via the Fourier transform. This technique is known as the Stolt migration and it is popular in Geophysics, see [29, 33. However, in the standard Stolt migration the wave at the initial time is calculated in the whole spatial domain of interest, whereas we calculate the wave only at a plane parallel to the measurement plane but in the whole time interval. This technique is described as follows. 
We assume that the scattered wave propagates in the positive $z$-direction. Denote by $P_{m}=$ $\{z=b\}, b>0$, the measurement plane and by $P_{p}=\{z=a\}$, with $a<b$, the propagated plane, which is closer to the target of interest than $P_{m}$. We also denote by $u^{s}(\mathbf{x}, t)$ the scattered wave. Our objective here is to determine the function $g(x, y, t):=u^{s}(x, y, a, t)$, given the measured data $f(x, y, t):=u^{s}(x, y, b, t)$. We assume that the medium is homogeneous in the half space $z \geq a$ with $\epsilon \equiv 1$. Therefore, $u^{s}$ is the solution of the following problem:

$$
\begin{aligned}
& u_{t t}^{s}-\Delta u^{s}=0, \mathbf{x} \in \mathbb{R}^{3}, z \geq a, t \in(0, \infty), \\
& u^{s}(\mathbf{x}, 0)=u_{t}^{s}(\mathbf{x}, 0)=0 .
\end{aligned}
$$

Consider the Fourier transform

$$
\hat{u}^{s}\left(k_{x}, k_{y}, z, \omega\right)=\int_{0}^{\infty} \int_{-\infty}^{\infty} \int_{-\infty}^{\infty} u^{s}(x, y, z, t) e^{-i\left(\omega t+x k_{x}+y k_{y}\right)} d x d y d t .
$$

It follows from (47) that $\hat{u}^{s}$ satisfies the equation:

$$
\begin{aligned}
\hat{u}_{z z}^{s}+\left(\omega^{2}-k_{x}^{2}-k_{y}^{2}\right) \hat{u}^{s} & =0, z \geq a, \\
\hat{u}\left(k_{x}, k_{y}, z, \omega\right) & =\hat{g}\left(k_{x}, k_{y}, \omega\right),
\end{aligned}
$$

where $\hat{g}\left(k_{x}, k_{y}, \omega\right)$ is the Fourier transform (49) of $g(x, y, t)$. We consider two cases:

Case 1: $\omega^{2}-k_{x}^{2}-k_{y}^{2}<0$. Keeping in mind that the scattered wave propagates in the positive $z$-direction, the problem (50)-(51) has the following solution

$$
\hat{u}_{1}^{s}\left(k_{x}, k_{y}, z, \omega\right)=\hat{g}\left(k_{x}, k_{y}, \omega\right) \exp \left(-(z-a) \sqrt{k_{x}^{2}+k_{y}^{2}-\omega^{2}}\right), z>a .
$$

Case 2: $\omega^{2}-k_{x}^{2}-k_{y}^{2} \geq 0$. Then the solution $\hat{u}^{s}$ can be represented as

$$
\hat{u}_{2}^{s}\left(k_{x}, k_{y}, z, \omega\right)=\hat{g}\left(k_{x}, k_{y}, \omega\right) \exp \left(-i(z-a) \sqrt{\omega^{2}-k_{x}^{2}-k_{y}^{2}}\right), z>a .
$$

The negative sign in the exponential term in this formula is due to the fact that the scattered wave is out-going in the positive $z$-direction.

Since the solution (52) is exponentially decaying as $z \rightarrow \infty$, which represents the evanescent wave, it practically cannot propagate to the measurement plane, which is in the far field zone. Hence,

$$
\hat{f}\left(k_{x}, k_{y}, \omega\right)=\hat{u}_{2}^{s}\left(k_{x}, k_{y}, b, \omega\right)=\hat{g}\left(k_{x}, k_{y}, \omega\right) \exp \left(-i(b-a) \sqrt{\omega^{2}-k_{x}^{2}-k_{y}^{2}}\right) .
$$

Using the inverse Fourier transform, we obtain

$$
g(x, y, t)=\iiint_{\omega^{2}-k_{x}^{2}-k_{y}^{2}>0} \hat{f}\left(k_{x}, k_{y}, \omega\right) e^{i(b-a) \sqrt{\omega^{2}-k_{x}^{2}-k_{y}^{2}}} e^{i\left(\omega t+x k_{x}+y k_{y}\right)} d k_{x} d k_{y} d \omega .
$$

Given the data $f(x, y, t)$ at the measurement plane, we compute $\hat{f}$ as well as $g(x, y, t)$ via (54) using the Fast Fourier Transform.

For each data set, the propagated plane $P_{p}$ was determined as follows. We first propagated the data to the sand's surface. Using this propagated data, we estimated the burial depths of 
the targets (section 4.2.2). Next, if the burial depth of the target closest to the sand surface was larger than $4 \mathrm{~cm}$, we propagated the data again from the measurement plane up to the plane $P_{p}$, whose distance to the front surface of that target was approximately $4 \mathrm{~cm}$. Otherwise, we used the data propagated up to the sand's surface for the next step of data preprocessing. Note that even we propagated the data beyond the sand's surface, we still saw the reflection from the sand's surface in the propagated data since we did not take into account the presence of the sand box in the data propagation, i.e., when propagating the data in the sand, we assumed that $\epsilon=1$ in the sand. This reflection from the sand's surface was removed when the targets' signals were extracted, see section 4.2.2. Note that the grid points at $P_{p}$ are the same as the ones at the measurement plane $P_{m}$. Thus, below we call "detectors" the grid points at the propagated plane $P_{p}$.

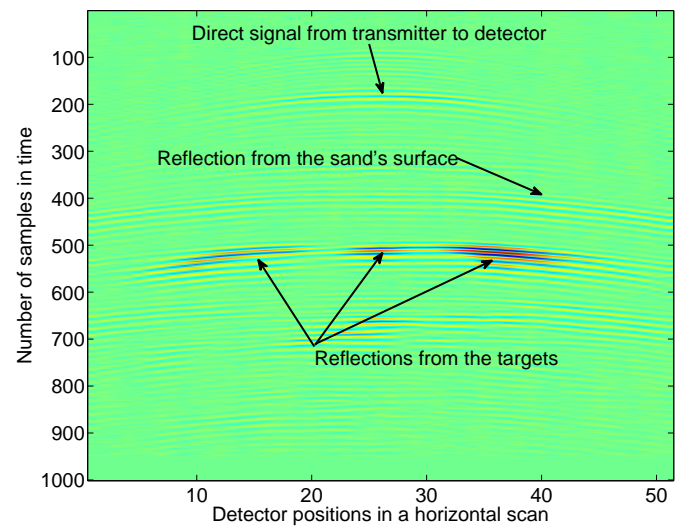

(a) Before propagation

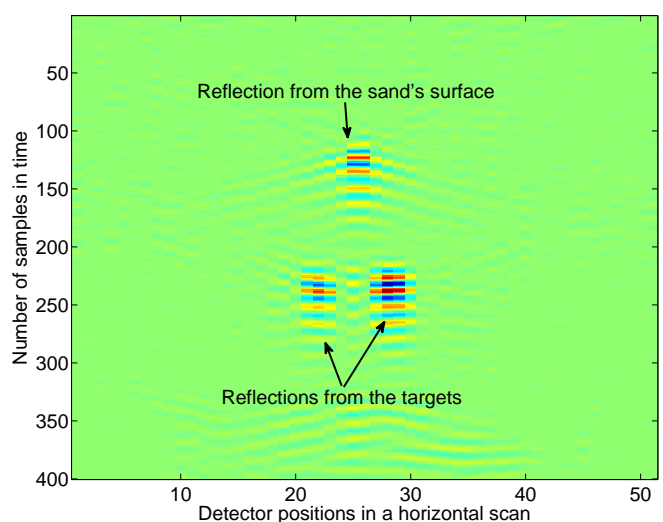

(b) After propagation

Figure 2: Result of the data propagation for signals from two targets buried inside the sand box. The signals of the two targets are well separated from each other as well as from the reflection from the sand's surface after the data propagation.

A result of the data propagation is illustrated in Figure 2, The figure shows a horizontal scan of the sand box containing two buried metallic targets. The horizontal side denotes the indices of the detector's locations and the vertical side denotes time. Time increases from the top to the bottom. The propagation distance, $b-a$, is $0.8 \mathrm{~m}$, which means that the propagated plane $P_{p}$ almost coincides with the sand's surface. Figure 2(a) shows the original data while Figure 2 (b) shows the data after the propagation. As can be seen from these figures, the targets' signals in the original data are smeared out. On the other hand, they are focused after the data propagation making the two targets more clearly distinguished. This is well known for migration methods. Moreover, we can also see that the reflection from the sand's surface is also more visible after the propagation and is well separated from the targets' signals.

\subsubsection{Estimation of the burial depth}

Since the sand's surface reflects our microwave pulses and these reflected waves arrive at the detectors before the ones reflected from the targets (see Figure 2(b)), the targets' signals are dominated by that of the sand's surface after the Laplace transform. In addition, the measure- 
ment noise appearing earlier than the targets' signals also affects the latter after the Laplace transform due to the exponential decay of the kernel, see Figure 4(a). Therefore, an additional important data preprocessing step is needed in order to pick up only the reflection from the targets and remove unwanted signals and noise coming earlier than the targets' signals. This step is applied to the propagated data as described below.

We first define some terms which are used in this section. These terms are related only to the propagated plane $P_{p}$. The strongest detector in a data set is defined as the detector at which the recorded signal has the largest amplitude. A strong target is either a metallic one or a nonmetallic one whose dielectric constant is larger than that of the sand. If the dielectric constant of a target is smaller than that of the sand, we call it a weak target. The strongest negative (positive) peak of a time-dependent signal at a certain detector's location is the negative (positive) peak whose amplitude is larger than the amplitudes of other negative (positive) peaks of the same signal.

We first estimate the burial depth of a target in each data set. For this purpose, we took the strongest detector. We first determined the strongest negative peak among the first four peaks, starting from the first negative peak, see Figure 3. This strongest negative peak is considered as the strongest negative peak of the sand's signal. After that, we excluded those first four peaks. The reason for considering those four peaks was due to our observation that those peaks should belong to the reflection from the sand's surface. Moreover, the first two negative (so as two positive) peaks of the incident wave were increasing in amplitude. After that, the negative (so as positive) peaks of the incident wave decreased in amplitude. Therefore any increase in amplitude of the peaks after those first four peaks should be due to the reflection from the target. By detecting the next negative (or positive) peak which was stronger than the previous negative (positive) one, we located the target's signal. Then, we determined the strongest negative peak of the target's signal. Denoting by $\Delta t$ the time delay between the latter peak and the strongest negative peak of the sand's signal, the burial depth of the target was approximated by $n$ (sand) $\Delta t$, where $n$ (sand) $=\sqrt{\epsilon(\operatorname{sand})}$ is the refractive index of the sand.

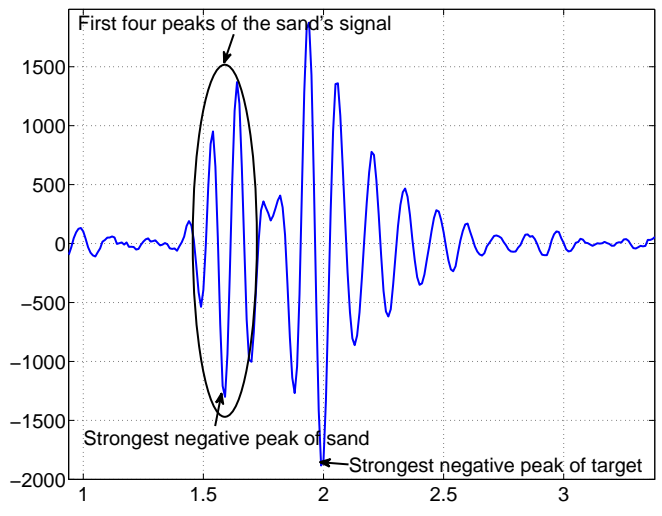

(a) A strong target

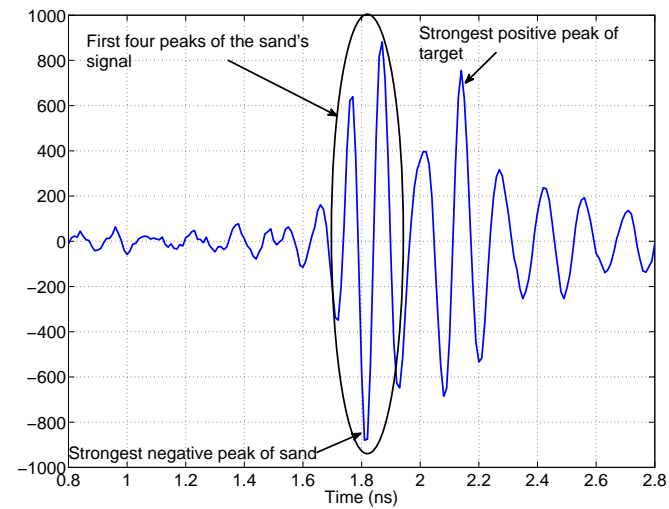

(b) A weak target

Figure 3: One-dimensional propagated signals at the strongest detectors of two targets: one strong target and one weak target. The signals consist of the reflection from the sand's surface followed by the reflections from the targets. 


\subsubsection{Extraction of target's signal: the most difficult step of data preprocessing}

After estimating the burial depth, we extracted the target's signal. The extraction of signals of targets in air is quite simple. However, it is very challenging in the case of buried objects, especially for weak targets. Indeed, when a weak target is buried at a shallow depth, its signal is merged with the reflection from the sand's surface. When it is buried at a deep depth, its signal is usually too weak to be visible in the data. Our experimental observations have shown that if a weak target is buried at a depth of more than $5 \mathrm{~cm}$, then we cannot detect it. In this case, the target is missed.

As in estimating the burial depth, we also worked with the strongest detector first and excluded the first four peaks. After that, we selected the target's signal as follows: (i) Suppose that either the burial depth was larger than $5 \mathrm{~cm}$, or the strongest negative peak of the target's signal was larger than that of the sand's signal in amplitude. Then we choose as the first peak of the target's signal the strongest negative peak located after the excluded ones; (ii) otherwise, the first peak of the target's signal was determined as the first positive peak which was larger than the previous positive one, provided that such a peak exists, see Figure 3. Since the reconstructed dielectric constants of targets of case (i) (respectively, case (ii)) was always larger (smaller) than that of the sand, we also categorized a target in case (i) (case (ii)) as a strong (weak) target. For all other detectors, we started from those closest to the strongest detector and on each of them assigned as the first peak of the target's signal the one which was time wise closest to that of the strongest detector. For strong targets this one should be a negative peak, while it was a positive peak for weak targets. Next, we continued similarly on all other detectors via sequentially choosing those peaks closest to the one of the previous detector. The reason for choosing a negative (positive) peak as the first peak of the target's signal for strong (weak) targets was due to our observations in numerical simulations and experimental data which have indicated that:

a. For a strong target, the first peak of the target's signal should be negative.

b. For a weak target, the first peak of the target's signal should be positive.

Moreover, if a strong target is buried at a depth less than $5 \mathrm{~cm}$, then its signal is stronger than that of the sand in amplitude. If the burial depth is more than $5 \mathrm{~cm}$, then its signal might not be stronger than that of the sand. However, since, as we mentioned above, weak targets are not visible at depths larger than $5 \mathrm{~cm}$, we consider all targets buried at these depths as strong targets.

In all above cases, the data before the chosen first peak of the target's signal were set to zero. Hence, the Laplace transform of the preprocessed data is not affected by values before the first chosen peak. We note that such a choice of starting peaks artificially immerses our targets in air: because we exclude the reflection from the sand's surface. Therefore, what we reconstruct for each target by the globally convergent method is the ratio between its dielectric constant (or the effective dielectric constant for metals) and that of the sand, $\epsilon$ (target) $/ \epsilon$ (sand). Next, to obtain the value of the dielectric constant of the target, we multiply this ratio by $\epsilon$ (sand) $=4$.

Figure 3 shows one-dimensional propagated signals at the strongest detectors of a strong target and a weak target. We indicate there the sand's signal and the peaks of the targets. These peaks were chosen as the first peaks of signals from the targets. Samples of the Laplace transform of the data before and after the extraction of the targets' signals are shown in Figure 4. which indicate the necessity of this preprocessing step.

Figure 4 (b) also shows that the preprocessed data allow us to estimate locations of the 
targets in $x, y$ directions as well as their $x y$-cross sections, see Section 4.3 of [30] for the method we proposed for estimating the cross sections of targets. These types of information help to reduce the domain in which we look for the targets. Indeed, in Test 2 below, we took into account these types of information in choosing the first tail function.

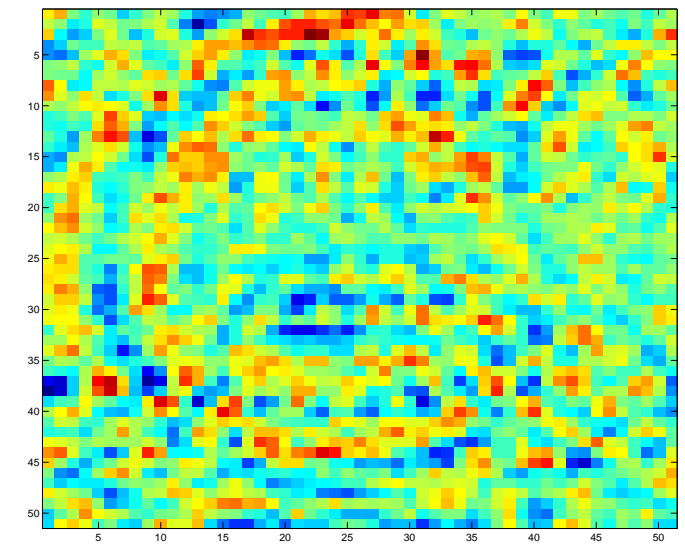

(a) Before the extraction of the targets' signals

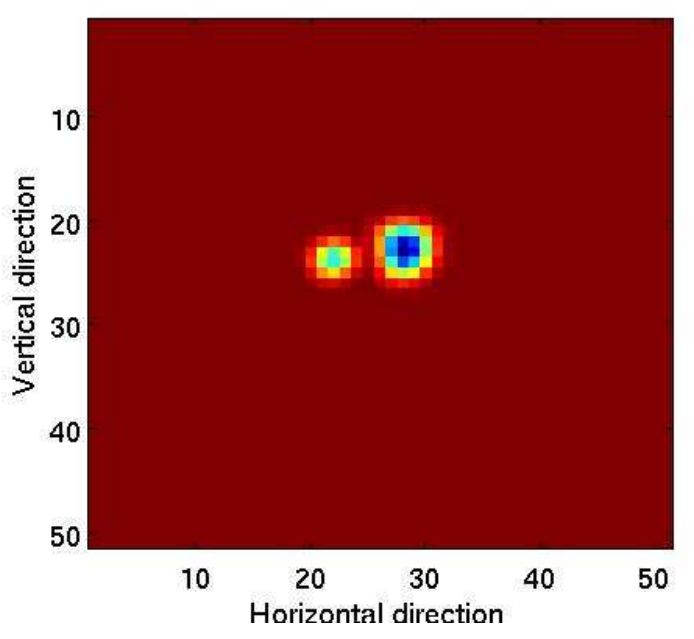

(b) After the extraction of the targets' signals

Figure 4: The Laplace transforms of the data on the propagated plane before (a) and after (b) the extraction of the targets' signals. Without the extraction, we cannot see the targets. After the extraction, the two targets show up clearly.

\section{$5 \quad$ Reconstruction results}

We now illustrate the performance of the globally convergent algorithm of Section 2.2 for our experimental data sets.

\subsection{Description of experimental data sets}

To have a rather systematic study of the performance of the globally convergent algorithm for these data, we have tested this algorithm for different types of targets at different burial depths varying between $0.02 \mathrm{~m}$ and $0.14 \mathrm{~m}$. Our results in this paper include 25 data sets. Table 1 describes the details of these data sets including the targets' materials. Among them, there are 10 non-blind cases and 15 blind ones. "Blind" means that the targets were unknown to the computational team (NTT, LB, MVK) but known to MAF, who was leading the data collection process. The non-blind targets are the ones that were known to NTT and MVK but were not known to LB who performed Test 1 below. Note that we were able to directly measure refractive indices $n=\sqrt{\epsilon}$ rather than dielectric constants. The refractive indices of all non metallic targets were measured after the reconstruction results were obtained by the computational team. Next, computational results were compared with directly measured ones. Some of the non-blind targets were used for calibrating and fine tuning of the reconstruction procedure. The blind targets were 
Table 1: Description of the test data sets. Seven of them consist of two targets each $(5,6,16,17,20$, 23, 25). Two targets can be considered as heterogeneous (11, 12).

\begin{tabular}{|c|c|c|c|}
\hline $\begin{array}{l}\text { Object } \\
\quad \#\end{array}$ & $\begin{array}{l}\text { Blind/ } \\
\text { Non-blind }\end{array}$ & Description of target & Material \\
\hline 1 & Non-blind & A metallic cylinder & Metal \\
\hline 2 & Non-blind & A metallic ball & Metal \\
\hline 3 & Non-blind & A bottle filled with clear water & Water \\
\hline 4 & Non-blind & A wet wooden block & Wet wood \\
\hline 5 & Non-blind & Two metallic blocks at $6 \mathrm{~cm}$ separation & Metal/Metal \\
\hline 6 & Non-blind & A metallic cylinder and a teflon bar & Metal/Teflon \\
\hline 7 & Non-blind & A metallic block & Metal \\
\hline 8 & Non-blind & An empty bottle & Air \\
\hline 9 & Non-blind & A bottle filled with teflon bars & Teflon \\
\hline 10 & Blind & A ceramic mug & Ceramic \\
\hline 11 & Blind & $\begin{array}{l}\text { A wooden doll filled with metallic screws } \\
\text { (heterogeneous, diffuse scattering) }\end{array}$ & Wood/Metal \\
\hline 12 & Blind & $\begin{array}{l}\text { A geode (heterogeneous): } \\
\text { two spherical layers and air inside }\end{array}$ & Rock \\
\hline 13 & Blind & A piece of rock & Rock \\
\hline 14 & Blind & A plastic bottle filled with coffee grounds & Coffee grounds \\
\hline 15 & Blind & A ceramic mug & Ceramic \\
\hline 16 & Blind & A cylinder and a block at $3 \mathrm{~cm}$ separation & Metal/Metal \\
\hline 17 & Blind & An aluminum can and a block & Metal/Metal \\
\hline 18 & Blind & $\begin{array}{l}\text { A wooden doll with a metallic block inside } \\
\text { (heterogeneous) }\end{array}$ & Wood/Metal \\
\hline 19 & Blind & A bottle of water & Water \\
\hline 20 & Blind & A metallic block and a rock & Metal/rock \\
\hline 21 & Blind & A steel mug & Metal \\
\hline 22 & Blind & A wet wooden block & Wet wood \\
\hline 23 & Blind & A wet wooden block and an empty bottle & Wet wood/air \\
\hline 24 & Blind & A wet wooden block & Wet wood \\
\hline 25 & Non-blind & Two metallic blocks at $1 \mathrm{~cm}$ separation & Metal/Metal \\
\hline
\end{tabular}

used to ensure that this procedure works in realistic blind data cases. It is important that the same reconstruction procedure, with the same choice of parameters, was used for all targets.

The burial depths of the targets varied between 2.5 centimeters $(\mathrm{cm})$ to $14 \mathrm{~cm}$. Note that typically burial depths of antipersonnel land mines do not exceed $10 \mathrm{~cm}$. Among our targets were metallic blocks, wooden blocks, metallic cylinders, metallic spheres, wooden dolls, etc. Seven data sets $(\# 5,6,16,17,2023,25)$ consisted of two targets each. In particular, there were three targets (\#11, 12, 18) which can be considered as heterogeneous ones, see Table 1. The data of the sand box alone (without buried objects) was used for the calibration of our data.

\subsection{Numerical implementation}

Details of the numerical implementation of the globally convergent algorithm can be found in [6, 30]. For the reader's convenience, we briefly describe the main points here.

Choosing the domain and solving the forward problem: In the preprocessed data, the distance from the front sides of the targets to the backscattering boundary of our inversion 
domain $\Omega$ was $0.04 \mathrm{~m}$. The reason for choosing this distance was due to good reconstruction results we had obtained for several non-blind targets. Hence, the inversion domain $\Omega$ was chosen as

$$
\Omega=\{\mathrm{x} \in(-0.4,0.4) \times(-0.4,0.4) \times(-0.2,0.04)\} .
$$

Moreover, since it is impossible to solve the problem (11)-(2) in the entire space, in numerical computation, we approximated it by an initial boundary value problem in a bounded domain $G \subset \mathbb{R}^{3}$ such that $\Omega \subset G$, see [6, 30] for details. In this paper, we choose $G$ as the rectangular parallelepiped

$$
G=\{\mathrm{x} \in(-0.5,0.5) \times(-0.5,0.5) \times(-0.3,0.3)\} .
$$

This domain $G$ was decomposed into two subdomain: $G=\Omega \cup(G \backslash \Omega)$. We recall that $\epsilon(\mathbf{x})=1$ in $G \backslash \Omega$. Therefore, it is only necessary to solve the inverse problem in $\Omega$. A finite element mesh with tetrahedral elements is used in $\Omega$, while in $G \backslash \Omega$ we use a finite difference mesh with mesh sizes of $0.02 \times 0.02 \times 0.02$. The forward problem was solved using the software package WavES [32] via a hybrid finite difference/finite element method described in [9].

The time interval on which the problem (11) -(2) was solved was chosen to be $(0, T)=(0,1.2)$ since no relevant signals appeared after this time interval. Note that the time variable was scaled so that the dielectric constant of air is equal to 1 . Since the explicit scheme in time was used in WaveES, the time step size was chosen as $\Delta t=0.0015$ which satisfied the CFL stability condition.

The pseudo frequencies $s_{n}$ were chosen from $\underline{s}=7$ to $\bar{s}=9$ with the step size $h=0.05$. This pseudo frequency interval was chosen because it gave good reconstructions of the non-blind targets. We have observed that the pseudo frequency interval $s \in[8,10]$ also provided good reconstruction results.

In our simulations, the waveform function $f$ in (11) was chosen by $f(t)=2 \omega \cos (\omega t)$ for $0 \leq t \leq t_{1}=2 \pi / \omega$, and $f(t)=0$ for $t>t_{1}$. Here, $\omega=30$ is the angular frequency of the incident plane wave.

Completing the backscattering data: We recall that our data are available only on the backscattering side of the inversion domain, i.e. at $\bar{\Omega} \cap\{z=0.04\}$. Therefore, the missing boundary data on the other sides of the rectangular parallelepiped $\Omega$ were approximated by the corresponding simulated data for the homogeneous medium with $\epsilon(\mathbf{x}) \equiv 1$ (we recall that after extracting the targets' signals, as explained in section 4.2.3, the targets were treated as ones placed in air). This approximation has been used in our previous works and found to provide good reconstruction results, see [7, 10, 30].

\subsection{Two tests and stopping criteria}

We have analyzed the performance of the proposed algorithm with two different tests: Test 1 and Test 2. In Test 1, we made use of the first tail function as described in section 2.3, with which the global convergence is rigorously guaranteed. In Test 2 , the estimated burial depth and the $x y$-cross section of the target via the data preprocessing procedure were used to restrict the domain in which the coefficient $\epsilon$ was reconstructed and to choose the first tail function. More precisely, for each target, let $x_{t, \min }=\min \left\{x \in \Gamma_{T}\right\}, \quad x_{t, \max }=\max \left\{x \in \Gamma_{T}\right\}$, where $\Gamma_{T}$ is the estimated $x y$ cross section of the target, see [30] for how this cross section is estimated. The numbers $y_{t, \min }$ and $y_{t, \max }$ are defined similarly. Then, we define the extended $x y$ cross section by

$$
\Gamma_{T, e x t}=\left\{x_{t, \min }-0.03<x<x_{t, \max }+0.03, y_{t, \min }-0.03<y<y_{t, \max }+0.03\right\} .
$$


Moreover, denote by $z_{t, \text { front }}$ the estimated location of the front side of the target in the $z$ direction, given by the burial depth estimation. We then define the following domain $\Omega_{T, e x t}$

$$
\Omega_{T, e x t}:=\left\{\mathbf{x} \in \Omega:(x, y) \in \Gamma_{T, e x t},-0.2<z<z_{t, \text { front }}+0.02\right\} .
$$

Clearly, $\Omega_{T, e x t} \subset \Omega$. Moreover, this domain should contain the unknown target we are looking for. The last number 0.02 was for compensating for possible error in the estimated burial depth of the target. Next, we chose the first tail function $V_{0}$ as the function (15), where the function $w(\mathbf{x}, \bar{s})$ was computed for the coefficient $\epsilon(\mathbf{x}):=\epsilon_{0}(\mathbf{x})$, where

$$
\epsilon_{0}(\mathbf{x})=\epsilon_{u}, \text { for } \mathbf{x} \in \Omega_{T, e x t}, \epsilon_{0}(\mathbf{x})=1 \text {, for } \mathbf{x} \notin \Omega_{T, e x t} .
$$

In this paper, the upper bound $\epsilon_{u}$ for the function $\epsilon(\mathbf{x})$ was chosen as $\epsilon_{u}=25$.

Although the convergence of the resulting algorithm for Test 2 has not been rigorously proved yet, our numerical results show good reconstructions, see also [30] for results when targets are in air. Note that we did not use a priori information about the targets. Instead, the information used in choosing the first tail function was derived from data preprocessing.

Stopping criteria: As mentioned in Remark 3.1. stopping criteria of the algorithm should be addressed numerically. In this paper, we used the stopping criteria proposed in [10, 30]. We briefly recall these criteria here for the reader's convenience.

Stopping criterion of Test 1: The inner iteration with respect to $i$ is stopped at $i=m_{n}$ such that

$$
D_{n, i} \geq D_{n, i-1}, \text { or } i<i_{\max }
$$

where $D_{n, i}=\left.|| V_{n, i}\right|_{\Gamma_{p}}-V_{\text {prop }}||_{L_{2}\left(\Gamma_{p}\right)}$. Here $\Gamma_{p}$ is the backscattering side of $\Omega$ and $V_{\text {prop }}$ is the tail function computed from the propagated data at $\Gamma_{p}$, and $i_{\max }$ is the maximum number of inner iterations. In Test 1 , we have chosen $i_{\max }=8$.

The outer iteration with respect to the pseudo frequency is stopped when the error function $D_{n, 1}$ attains the first local minimum with respect to $n$.

Stopping criterion of Test 2: The inner iteration is stopped using the same criterion as in Test 1 but with $i_{\max }=5$. The outer iteration is stopped when the error function $D_{n, m_{n}}$, i.e., the error function at the final inner iteration, attains the first local minimum.

We have observed in our tests that these stopping criteria gave good results for non blind targets.

\subsection{Summary of reconstruction results and discussion}

In Tables 2 and 3 we summarize reconstruction results of the two tests for the data sets listed in Table 1. Table 2 shows the results for the non metallic targets. For these targets, the refractive index $n$ (target) $=\sqrt{\epsilon \text { (target) }}\left(\epsilon\right.$ (target) was chosen by $\epsilon($ target $\left.)=\max _{\mathbf{x} \in \Omega} \epsilon(\mathbf{x})\right)$ is shown instead of the dielectric constant $\epsilon$ because $n$ (target) was directly measured after computations were performed. Table 3 shows the burial depths and the effective dielectric constants of the metallic targets.

As described in section 4.2.2, the burial depth was estimated based on the time delay between the reflection by the sand's surface and the target's signal. Note that our incident signal was not really a short impulse. It is therefore natural to expect some level of error in our estimates. Since we made use of peaks of the signals in estimating the depth, the error we expect is about the distance between two consecutive peaks, which is equal to half of the wavelength $(2 \mathrm{~cm})$. 
Table 2: Result of the globally convergent algorithm: the refractive indices $n=\sqrt{\epsilon}$ and the burial depths of non-metallic targets. Object \#11 is a heterogeneous target with diffuse scattering, see below. Object \#12 is a heterogeneous one with outer and inner layers, the computed $n$ is compared with the average measured $n=1.28$. Object \#23 consists of two targets: wet wood and empty bottle filled with air. "Comp." stands for "Computed". The average error of strong targets is $8.5 \%$ for Test 1 and $14.7 \%$ for Test 2. The average error of weak targets is 21.6\% for Test 1 and 13\% for Test 2.

\begin{tabular}{|c|l|c|c|l|l|l|}
\hline $\begin{array}{c}\text { Object } \\
\#\end{array}$ & Material & $\begin{array}{r}\text { Comp. } \\
\text { depth }\end{array}$ & $\begin{array}{c}\text { Exact } \\
\text { depth }\end{array}$ & $\begin{array}{l}\text { Comp. } \\
n, \text { Test 1 }\end{array}$ & $\begin{array}{l}\text { Comp. } \\
n, \text { Test 2 }\end{array}$ & $\begin{array}{l}\text { Measured } \\
n\end{array}$ \\
\hline 3 & Water & $3.6 \mathrm{~cm}$ & $4.0 \mathrm{~cm}$ & 4.7 & 4.9 & 4.88 \\
\hline 4 & Wet wood & $5.5 \mathrm{~cm}$ & $9.8 \mathrm{~cm}$ & 4.4 & 4.5 & 4.02 \\
\hline 8 & Air & $2.8 \mathrm{~cm}$ & $3.0 \mathrm{~cm}$ & 1.0 & 0.98 & 1.0 \\
\hline 9 & Teflon & $2.9 \mathrm{~cm}$ & $2.5 \mathrm{~cm}$ & 1.0 & 1.18 & 1.0 \\
\hline 10 & Ceramic & $4.0 \mathrm{~cm}$ & $5.0 \mathrm{~cm}$ & 1.0 & 1.23 & 1.39 \\
\hline 11 & Wood with & $4.6 \mathrm{~cm}$ & $4.0 \mathrm{~cm}$ & 1.0 & 1.46 & $\begin{array}{l}1.89 \text { (wood) } \\
\text { Netal screws }\end{array}$ \\
& & & & 1.3 diffuse scattering \\
\hline 12 & Geode & $2.1 \mathrm{~cm}$ & $2.5 \mathrm{~cm}$ & 1.0 & 1.52 & 1.31 (outer) \\
& (two layers) & & & & & 1.28 (average) \\
\hline 13 & Rock & $2.0 \mathrm{~cm}$ & $2.3 \mathrm{~cm}$ & 1.0 & 1.34 & 1.34 \\
\hline 14 & Coffee grounds & $2.0 \mathrm{~cm}$ & $2.5 \mathrm{~cm}$ & 1.0 & 1.46 & 1.11 \\
\hline 15 & Ceramic & $2.6 \mathrm{~cm}$ & $2.5 \mathrm{~cm}$ & 1.0 & 1.51 & 1.39 \\
\hline 19 & Water & $7.5 \mathrm{~cm}$ & $9.5 \mathrm{~cm}$ & 4.5 & 5.2 & 4.88 \\
\hline 22 & Wet wood & $2.9 \mathrm{~cm}$ & $3.0 \mathrm{~cm}$ & 4.8 & 5.3 & 4.02 \\
\hline 23 & Wet wood & $5.7 \mathrm{~cm}$ & $7.5 \mathrm{~cm}$ & 4.0 & 4.1 & 4.02 \\
& Empty bottle (air) & missed & $7.5 \mathrm{~cm}$ & missed & missed & 1.0 \\
\hline 24 & Wet wood & $5.1 \mathrm{~cm}$ & $6.8 \mathrm{~cm}$ & 3.67 & 3.0 & 4.02 \\
\hline
\end{tabular}


Table 3: Result of the globally convergent algorithm: the estimated effective dielectric constants and the burial depths of metallic targets. Object \#18 is a heterogeneous one: a wooden dool with a metallic block inside. Object \#20 consists of two targets: a metallic block and a rock. Measured $n($ rock $)=1.34$. Objects \#5, 16, 17 consist of two metallic targets. Object \#25 consists of two metallic targets with $1 \mathrm{~cm}$ distance between their surfaces: super resolution, see Figure 5.4 .

\begin{tabular}{|c|l|r|r|l|l|}
\hline $\begin{array}{c}\text { Object } \\
\#\end{array}$ & Material & $\begin{array}{r}\text { Computed } \\
\text { depth }\end{array}$ & $\begin{array}{r}\text { Exact } \\
\text { depth }\end{array}$ & $\begin{array}{l}\text { Computed } \epsilon \\
\text { Test 1 }\end{array}$ & $\begin{array}{l}\text { Computed } \epsilon \\
\text { Test 2 }\end{array}$ \\
\hline 1 & Metal & $2.9 \mathrm{~cm}$ & $4.0 \mathrm{~cm}$ & 29.9 & 46.4 \\
\hline 2 & Metal & $2.9 \mathrm{~cm}$ & $3.0 \mathrm{~cm}$ & 24.5 & 31.0 \\
\hline 5 & Metal & $3.0 \mathrm{~cm}$ & $3.0 \mathrm{~cm}$ & 23.4 & 32.4 \\
& Metal & $3.6 \mathrm{~cm}$ & $3.0 \mathrm{~cm}$ & 30.5 & 41.2 \\
\hline 6 & Metal & $2.8 \mathrm{~cm}$ & $8.5 \mathrm{~cm}$ & 27.8 & 37.5 \\
& Teflon & missed & $8.5 \mathrm{~cm}$ & & \\
\hline 7 & Metal & $9.9 \mathrm{~cm}$ & $14.0 \mathrm{~cm}$ & 47.4 & 65.8 \\
\hline 16 & Metal & $2.5 \mathrm{~cm}$ & $4.5 \mathrm{~cm}$ & 19.9 & 24 \\
& Metal & $3.7 \mathrm{~cm}$ & $4.5 \mathrm{~cm}$ & 33.7 & 47.5 \\
\hline 17 & Metal & $2.0 \mathrm{~cm}$ & $3.8 \mathrm{~cm}$ & 30.0 & 51.1 \\
& Metal & $2.7 \mathrm{~cm}$ & $3.8 \mathrm{~cm}$ & 54.8 & 93.5 \\
\hline 18 & Wood, metal block inside & $7.1 \mathrm{~cm}$ & $8.5 \mathrm{~cm}$ & 18.3 & 19.9 \\
\hline 20 & Metal & $6.8 \mathrm{~cm}$ & $8.5 \mathrm{~cm}$ & 30.0 & 48.1 \\
& Rock & missed & $8.5 \mathrm{~cm}$ & & \\
\hline 21 & Metal & $5.1 \mathrm{~cm}$ & $7.5 \mathrm{~cm}$ & 22.1 & 28.2 \\
\hline 25 & Metal & $3.8 \mathrm{~cm}$ & $4.0 \mathrm{~cm}$ & 70.0 & 99.8 \\
& Metal & $4.0 \mathrm{~cm}$ & $4.0 \mathrm{~cm}$ & 40.8 & 56.5 \\
\hline
\end{tabular}


From Tables 2 and 3 we can see that the burial depth was accurately estimated in most cases, with the errors not exceeding $2 \mathrm{~cm}$. There are two cases $(\# 4,7)$ in which the errors were about $4 \mathrm{~cm}$. These targets were buried at rather deep depths of about the limiting depth $(10 \mathrm{~cm})$ for antipersonnel land mines. This made the estimate less accurate because of possible uncertainty in measuring the refractive index of the sand. Also, there might be an error in recording the exact burial depths during the data acquisition for deeply buried targets.

The estimates of the refractive indices of non-metallic targets with refractive indices larger than that of the sand (water and wet wood) are quite accurate with the average error of about $9.7 \%$ for Test 1 and $15.2 \%$ for Test 2 . Note that the error in our direct measurement of the refractive index of the wet wood was $10 \%$. For water, we were unable to directly measure its refractive index at the used quite high frequency of the signal, which was about $7.5 \mathrm{GHz}$. Therefore, we have made a separate experiment: we have placed that bottle of water in air, measured the backscattering data and then reconstructed the refractive index using the globally convergent method as in [10, 30]. The result of $n=4.88$ matches well the experimentally measured refractive index of 4.84 at high frequencies in Table 3.1 of [15]. Moreover, by comparing our computed $n$ for water in Table 2 with this reference value $n=4.88$, we can see the consistency of our results.

Targets with smaller refractive indices than that of the sand are of interest since they are models of plastic land mines and IEDs. We have observed that we can image these targets only if their burial depths do not exceed $5 \mathrm{~cm}$. The average error shown in Table 2 for these weak targets is about $21.6 \%$ for Test 1 and $13 \%$ for Test 2 . The average measurement error of $n$ for weak targets was about $5.4 \%$.

In our experiments, we have missed some weak targets (not shown here), which had more than $5 \mathrm{~cm}$ burial depths. For these weak targets, we have observed that their signals were blended by the reflection from the sand's surface. Therefore, we could not detect any target's signal out of them. Note that, since our current algorithm uses the Laplace transform, it is applicable only when we can detect and extract targets' signals and remove the reflection by the sand's surface as well as noise at earlier times. Otherwise, they will dominate the targets' signals after the Laplace transform, see Figure 4. Thus, these missed cases were not due to the inversion algorithm.

The signals of the metallic targets were strong compared to the sand's signal. Therefore they were quite easy to detect. We recall that metallic targets can be approximated by dielectric ones with large effective dielectric constants. The estimated effective dielectric constants of our metallic test targets are between 20 and 100. In our previous works, we have established that the effective dielectric constant of metals should be larger than 10-15, see [10, 20, 30]. Therefore our results here are compatible with our previous studies.

In our tests, there were four cases (\#5,16, 17, 25 in Table 3) in which there were two metallic targets simultaneously. In each case we have accurately imaged both targets. In particular, in the data set \#25, the two metallic blocks were at $1 \mathrm{~cm}$ distance, see Figure 8 . On the other hand, the wavelength of our device is about $4 \mathrm{~cm}$. Thus, the super resolution is achieved, which is of about $\lambda / 4$, where $\lambda$ is the wavelength of our incident wave. This is an unexpected surprise. From a purely angular spectrum argument, the spread of backscatter angles for a fixed frequency would suggest a resolution of half a wavelength. However, there has been previous evidence reported that using a nonlinear inverse scattering algorithm for which strong or multiple scattering occurs, that some degree of super resolution (i.e., beyond the ideal diffraction limit of half a wavelength) can occur, see, e.g., [27]. This should be studied further. 


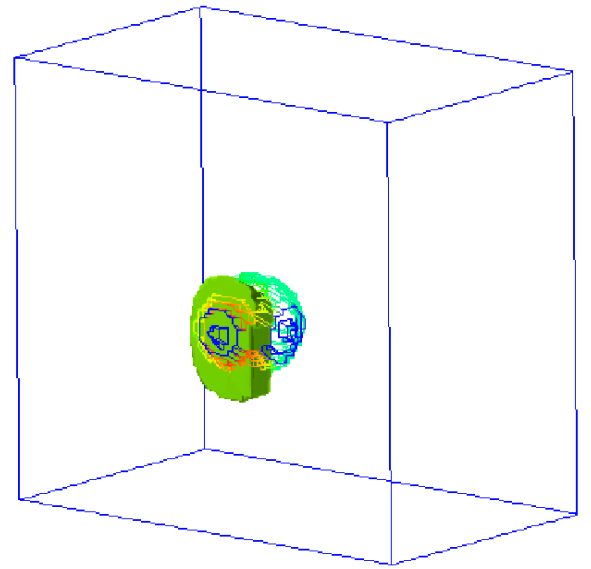

(a) Test 1, 3D view

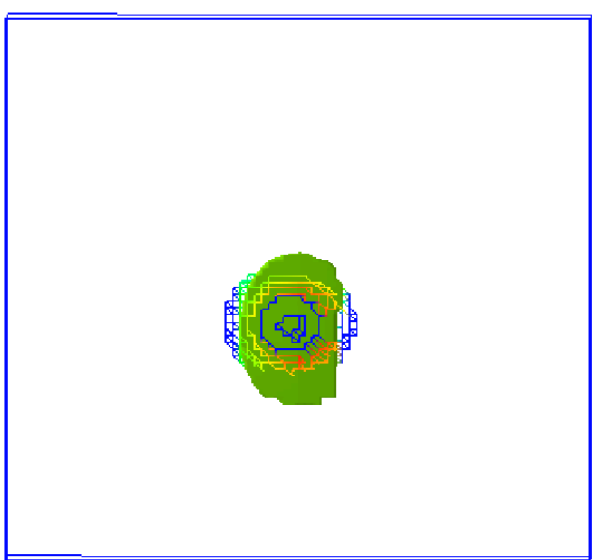

(c) Test $1, x y$ view

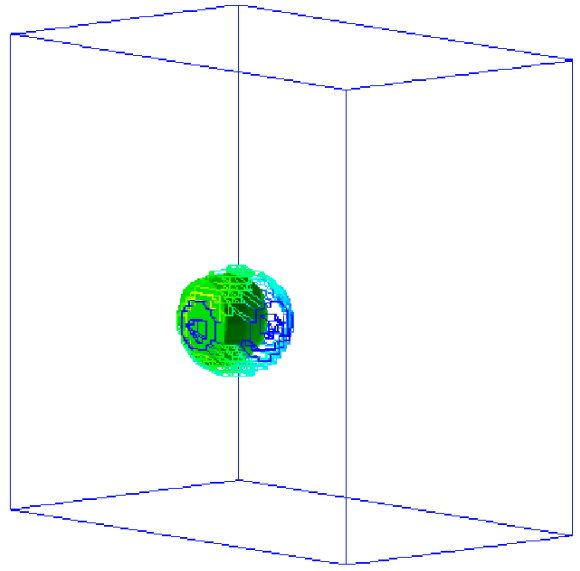

(b) Test 2,3D view

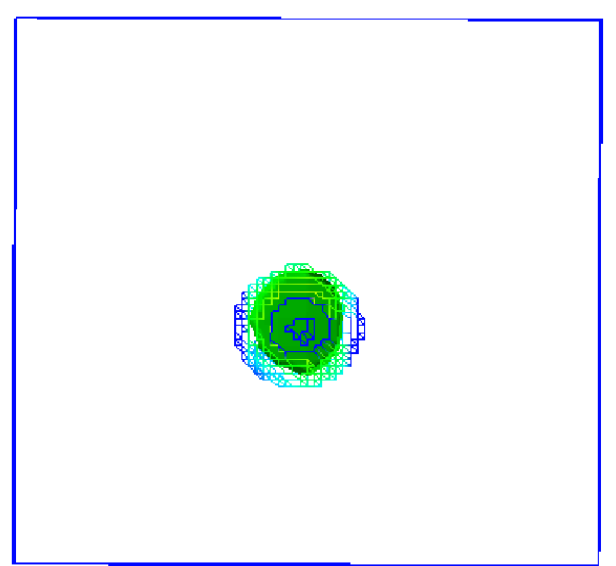

(d) Test 2, $x y$ view

Figure 5: Reconstructed shape of Target \#2 (a metallic ball): $x y$ view means the projections of the target on the $x y$ plane. The thin lines indicate the true shape. 


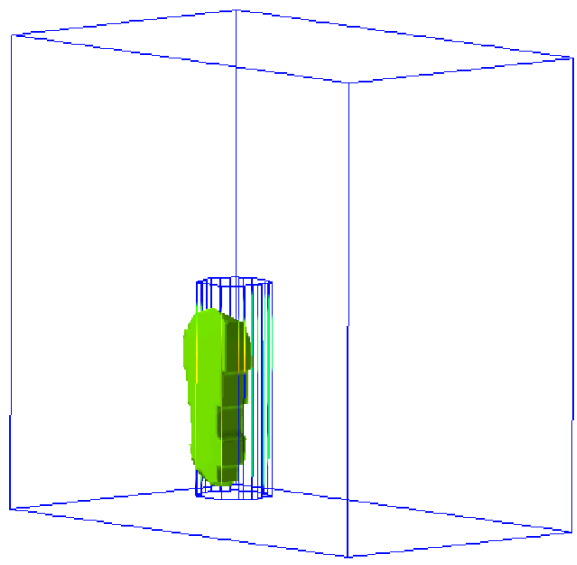

(a) Test $1,3 \mathrm{D}$ view

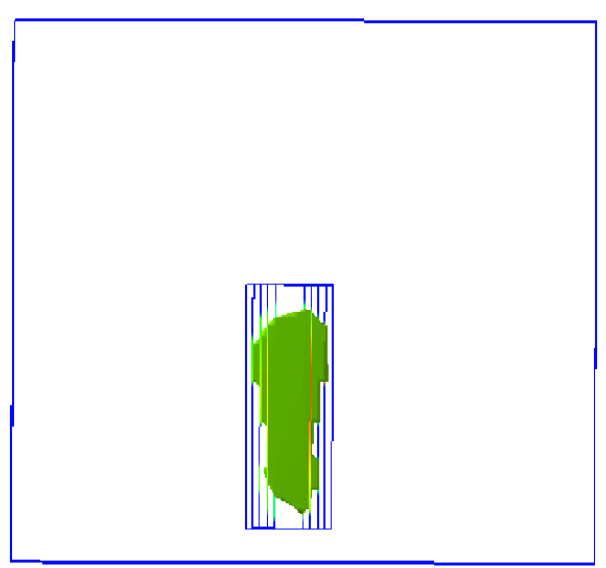

(c) Test 1, $x y$ view

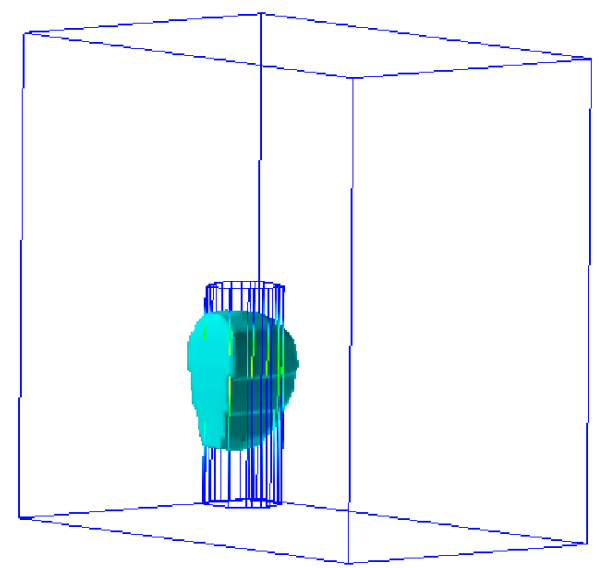

(b) Test 2,3D view

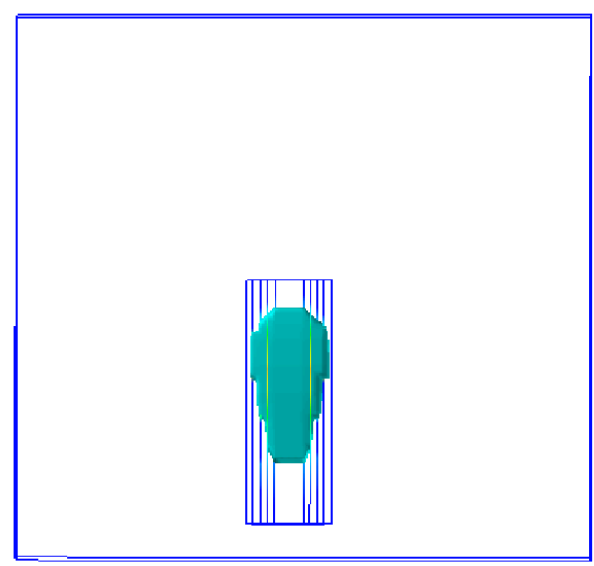

(d) Test 2, $x y$ view

Figure 6: Reconstructed shape of Target \#3 (a bottle of water). The thin lines indicate the true shape. 


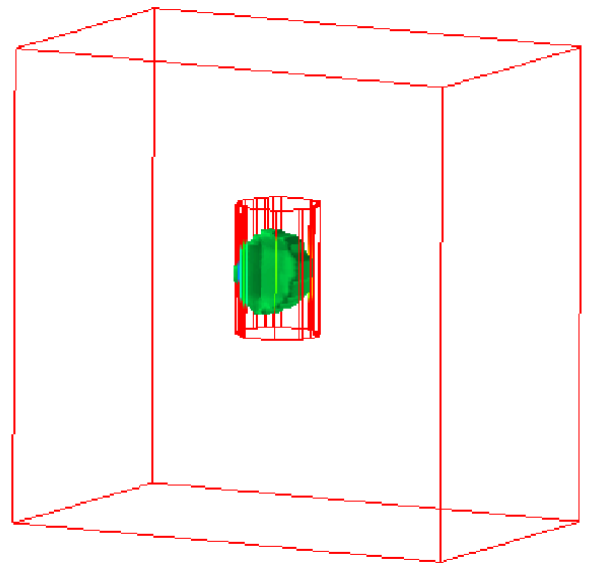

(a) Test 1, 3D view

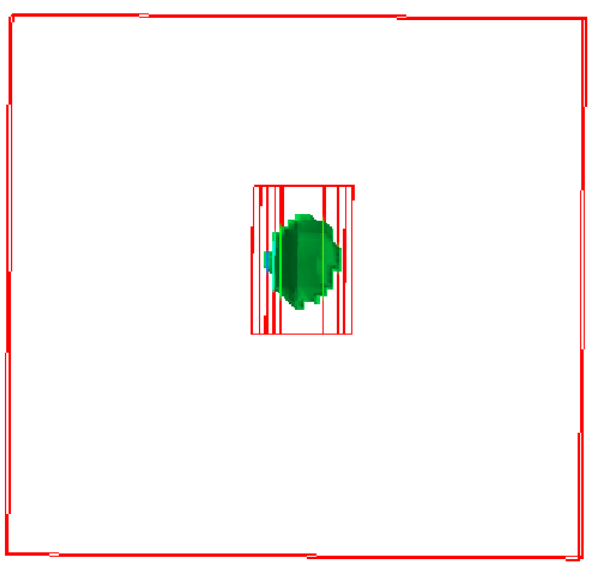

(c) Test $1, x y$ view

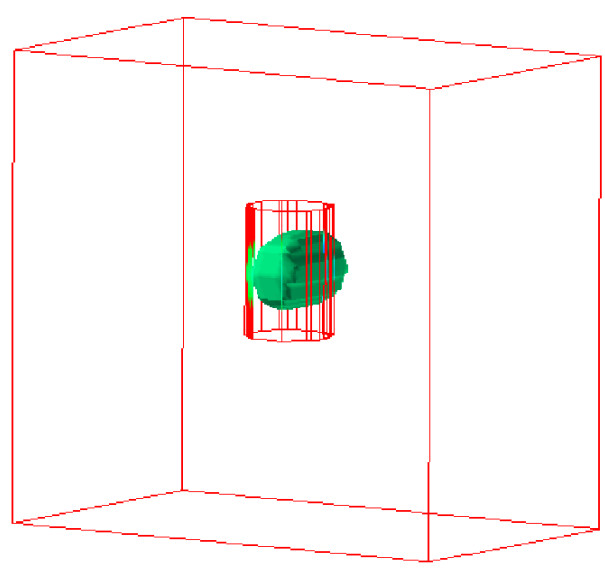

(b) Test 2,3D view

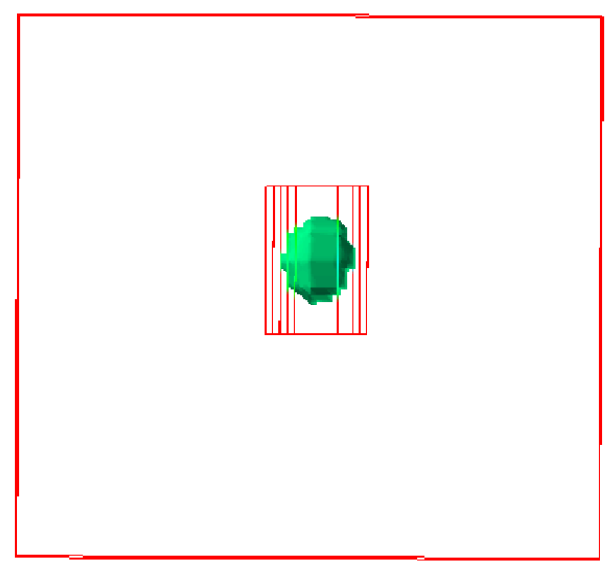

(d) Test 2, $x y$ view

Figure 7: Reconstructed shape of Target \#10 (a ceramic mug). The thin lines indicate the true shape. 


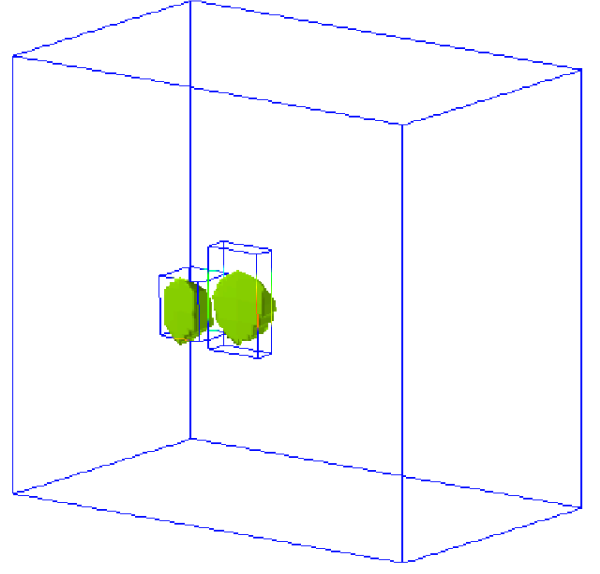

(a) Test 1, 3D view

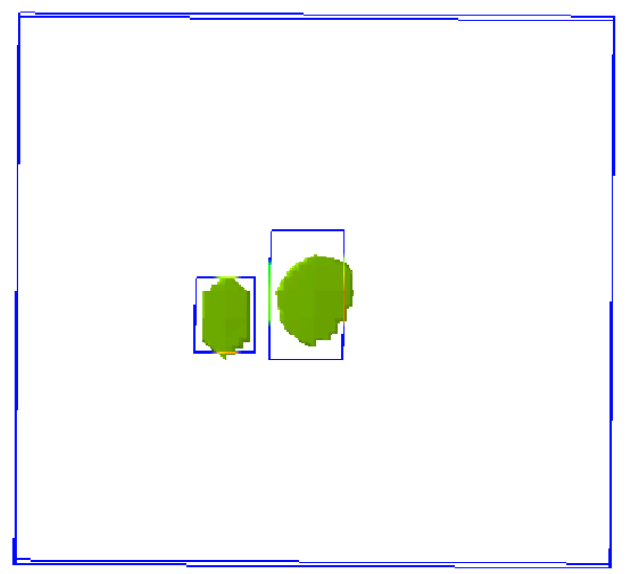

(c) Test $1, x y$ view

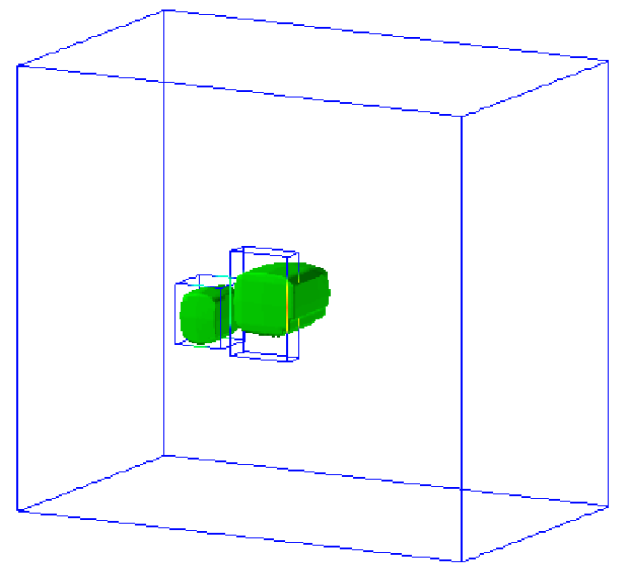

(b) Test 2,3D view

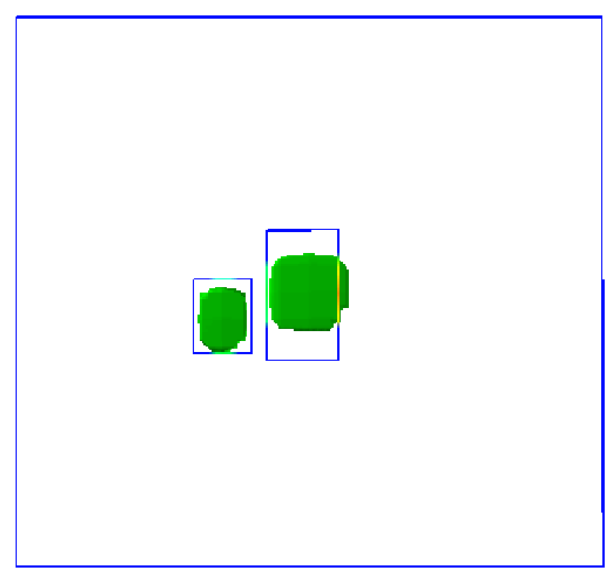

(d) Test 2, $x y$ view

Figure 8: Reconstructed shape of Target \#25 (two metallic blocks at $1 \mathrm{~cm}$ separation). The thin lines indicate the true shape. 
There were also three data sets (\#23 in Table 2 and \#6, 20 in Table 3) in which one strong target and one weak target were buried simultaneously. In all these three cases we have accurately imaged the stronger target. However, we missed the weaker ones. The reason why we missed the weak targets was due to the fact that their burial depths were larger than $5 \mathrm{~cm}$, which is our limiting depth for weak targets.

Also of interest are three cases of heterogeneous targets (\#11, 12 in Table 2 and \#18 in Table 3), since explosive devices are heterogeneous sometimes. We successfully estimated the average refractive index of the geode, which consists of two different layers, in data set \#12. For the wooden doll containing a metallic block inside in data set \#18 the computed dielectric constant is larger than that of the wood but smaller than other metallic targets. It is smaller because the wood covers the metal. Target \#11 was a wooden doll with randomly distributed metal screws inside. In this case we observed a weak signal, rather than a strong one from the metal. In fact, we observed a well known phenomenon of diffuse scattering, which was described in [26]. This can be explained since the metal screws were randomly oriented and represent a conducting very rough surface to the incident microwave pulse. Multiple scattering combined with the penetration of the microwaves into the gaps between the screws strongly attenuates the incident wave and little scatters back to contribute to a measurable signal.

The reconstruction of shapes of the targets is illustrated in Figures 5 8, For a better visualization, we show in these figures the targets in the box with sizes $0.4 \times 0.4 \times 0.24$, which is smaller in $x, y$ directions than our computational domain $\Omega$. To show the shapes of the targets, we computed a truncated coefficient $\epsilon_{t}(x, y, z)$ as follows:

$$
\epsilon_{t}(x, y, z)=\left\{\begin{array}{l}
\epsilon(x, y, z) \text { if }(x, y) \in \Gamma_{T} \text { and } \epsilon(\mathbf{x})>\gamma \epsilon_{\max } \\
\epsilon(\text { sand }) \text { otherwise }
\end{array}\right.
$$

for strong targets, with $\epsilon_{\max }$ is the maximum of $\epsilon(\mathbf{x})$. For weak targets, $\epsilon_{t}(x, y, z)$ is given by

$$
\epsilon_{t}(x, y, z)=\left\{\begin{array}{l}
\epsilon(x, y, z) \text { if }(x, y) \in \Gamma_{T} \text { and } \epsilon(\mathbf{x})<\gamma \epsilon_{\text {min }} \\
\epsilon(\text { sand }) \text { otherwise }
\end{array}\right.
$$

where $\epsilon_{\min }$ is the minimum value of $\epsilon(\mathbf{x})$. Recall that $\Gamma_{T}$ is the estimated $x y$ cross-section of the target, see section 5.3. In this paper, the truncation parameter $\gamma$ was chosen $\gamma=0.7$ for Test 1 and $\gamma=0.6$ for Test 2 .

Figure 5 depicts the reconstruction of target \#2 (a metallic ball buried at $3 \mathrm{~cm} \mathrm{depth).} \mathrm{We}$ can see that the shape is quite well reconstructed in Test 2 , especially the $x y$-cross section, since the measured data was acquired in the $x, y$-plane. Figure 6 shows the reconstruction of target \#3 (a plastic bottle filled with a clean water). In this case, since the target was quite high (about $18 \mathrm{~cm}$ ), the incident wave was weak at the vertical ends of the bottle which made it difficult to reconstruct the complete shape. However, we still can see in both tests the stretch in the vertical direction following the shape of the bottle. Figure 7 illustrates the reconstruction of target \#10, a ceramic mug. This is a weak target. We can see that its shape is less accurately reconstructed than ones of strong targets, since its signal is weak. Finally, Figure 8 shows the reconstruction of the most difficult case of data set \#25: the two targets at $1 \mathrm{~cm}$ separation. This seems to be a super resolution case as we discussed above. 


\section{Conclusions}

We have demonstrated the performance of the globally convergent algorithm of [6] for twenty five (25) test objects buried inside a sand box, which models imaging of subsurface objects. Since the signal from the sand is mixed with the signal from the target, this case is much harder than our previous results for targets placed in air [10, 30]. Our results have shown that it is possible to image refractive indices of non metallic and effective dielectric constants of metallic buried objects using backscattering time dependent measurements associated with only one incident wave. In general, the reconstruction results presented here are quite accurate. In particular, we have shown that the technique of [6] can image quite high target/background contrasts in dielectric constants (Table 3), which is usually hard to achieve by locally convergent algorithms. There are some cases in which weak targets were missed when they were in placed together with strong targets. This was due to the fact that their signals were too weak to be detected since they were buried at deep depths exceeding our limiting depth of $5 \mathrm{~cm}$. This made us unable to extract their signals.

To improve the accuracy of reconstruction of shapes of targets, we plan to use the adaptivity technique on the second stage of our two-stage numerical procedure, see for results for targets in air [11]. We also plan to generalize the current globally convergent inversion algorithm to the case of the frequency domain data in which the extraction of the target's signal will no longer be needed. This might lead to a lesser number of missed weak targets.

\section{Acknowledgment}

This research was supported by US Army Research Laboratory and US Army Research Office grants W911NF-11-1-0325 and W911NF-11-1-0399, the Swedish Research Council, the Swedish Foundation for Strategic Research (SSF) through the Gothenburg Mathematical Modelling Centre (GMMC) and by the Swedish Institute, Visby Program.

The authors are grateful to Mr. Steven Kitchin for his excellent work on data collection.

\section{References}

[1] A. B. Bakushinsky and M. Y. Kokurin. Iterative methods for approximate solution of inverse problems. Springer, Dordrecht, 2004.

[2] H. Bateman and A. Erdélyi. Tables of integral transforms. Vol 1. McGraw-Hill Book Company, Inc., New York, 1954.

[3] L. Beilina. Energy estimates and numerical verification of the stabilized domain decomposition finite element/finite difference approach for the maxwell's system in time domain. Central European Journal of Mathematics, 11(4):702-733, 2013.

[4] L. Beilina and M. V. Klibanov. A globally convergent numerical method for a coefficient inverse problem. SIAM Journal on Scientific Computing, 31(1):382-402, 2008.

[5] L. Beilina and M. V. Klibanov. Reconstruction of dielectrics from experimental data via a hybrid globally convergent/adaptive inverse algorithm. Inverse Problems, 26(12):125009, 30,2010 . 
[6] L. Beilina and M. V. Klibanov. Approximate Global Convergence and Adaptivity for Coefficient Inverse Problems. Springer, New York, 2012.

[7] L. Beilina and M. V. Klibanov. A new approximate mathematical model for global convergence for a coefficient inverse problem with backscattering data. Journal of Inverse and Ill-posed Problems, 20(4):513-565, 2012.

[8] L. Beilina and M. V. Klibanov. Relaxation property for the adaptivity for ill-posed problems. Applicable Analysis, 2013. DOI:10.1080/00036811.2013.768339.

[9] L. Beilina, K. Samuelsson, and K. Åhlander. Efficiency of a hybrid method for the wave equation. In International Conference on Finite Element Methods, Gakuto International Series Mathematical Sciences and Applications,. Gakkotosho CO., LTD, 2001.

[10] L. Beilina, N. T. Thành, M. Klibanov, and M. A. Fiddy. Finite elemement method in a quantitative imaging from experimental data using a globally convergent algorithm. Inverse Problems, 30(2):025002, 2014.

[11] L. Beilina, N. T. Thành, M. Klibanov, and J. B. Malmberg. Reconstruction of shapes and refractive indices from blind backscattering experimental data using the adaptivity. 2014. Preprint, arXiv:1404.5862 [math.NA].

[12] A. Bukhgeim and M. Klibanov. Uniqueness in the large of a class of multidimensional inverse problems. Soviet Math. Doklady, 17:244-247, 1981.

[13] G. Chavent. Nonlinear least squares for inverse problems. Theoretical foundations and step-by-step guide for applications. Springer, New York, 2009.

[14] H. W. Engl, M. Hanke, and A. Neubauer. Regularization of inverse problems. Kluwer Academic Publishers Group, Dordrecht, 1996.

[15] E. Farr and C. Frost. Impulse propagation measurements of dielectric properties of water, dry sand, moist sand and concrete. 1997. Measurement Note 52. Farr Research.

[16] A. Friedman. Partial differential equations of parabolic type. Prentice-Hall, Inc., Englewood Cliffs, N.J., 1964.

[17] D. Gilbarg and N. S. Trudinger. Elliptic partial differential equations of second order, volume 224 of Grundlehren der Mathematischen Wissenschaften [Fundamental Principles of Mathematical Sciences]. Springer-Verlag, Berlin, second edition, 1983.

[18] M. V. Klibanov, M. A. Fiddy, L. Beilina, N. Pantong, and J. Schenk. Picosecond scale experimental verification of a globally convergent algorithm for a coefficient inverse problem. Inverse Problems, 26(4):045003, 30, 2010.

[19] M. V. Klibanov and A. Timonov. Carleman estimates for coefficient inverse problems and numerical applications. Inverse and Ill-posed Problems Series. VSP, Utrecht, 2004.

[20] A. Kuzhuget, L. Beilina, M. Klibanov, A. Sullivan, L. Nguyen, and M. Fiddy. Blind backscattering experimental data collected in the field and an approximately globally convergent inverse algorithm. Inverse Problems, 28(9):095007, 33, 2012. 
[21] O. A. Ladyzhenskaya, V. A. Solonnilov, and N. N. Uralceva. Linear and Quasilinear Equations of Parabolic Types. American Mathematical Society, Providence, R.I., 1968.

[22] Y. Liu, J. Su, Z.-J. Lin, S. Teng, A. Rhoden, N. Pantong, and H. Liu. Reconstructions for continuous-wave diffuse optical tomography by a globally convergent method. 2013. Preprint, available online at http://www.ma.utexas.edu/mp_arc/, preprint number 13 - 87.

[23] M. Pastorino. Microwave Imaging. John Wiley \& Sons Inc., Hoboken, New Jersey, 2010.

[24] V. G. Romanov. Inverse Problems of Mathematical Physics. VNU, Utrecht, The Netherlands., 1986.

[25] V. G. Romanov. Stability estimates in inverse problems for hyperbolic equations. Milan J. Math., 74:357-385, 2006.

[26] A. Sentenac, H. Giovannini, and M. Saillard. Scattering from rough inhomogeneous media: splitting of surface and volume scattering. J. Opt. Soc. Am. A, 19(4):727-736, 2002.

[27] F. Simonetti. Localization of pointlike scatterers in solids with subwavelength resolution. Applied Physics Letter, 89:094105, 2006.

[28] M. Soumekh. Synthetic aperture radar signal processing. John Wiley \& Son, New York, 1999.

[29] R. Stolt. Migration by fourier transform. Geophysics, 43:23-48, 1978.

[30] N. T. Thành, L. Beilina, M. V. Klibanov, and M. A. Fiddy. Reconstruction of the refractive index from experimental backscattering data using a globally convergent inverse method. SIAM Journal on Scientific Computing, 36(3):B273-293, 2014.

[31] V. S. Vladimirov. Equations of mathematical physics. Translated from the Russian by Audrey Littlewood. Marcel Dekker, Inc., New York, 1971.

[32] WavES. The software package. Available online at http://waves24.com.

[33] O. Yilmaz. Seismic Data Imaging. Society of Exploration Geophysicists, Tulsa Oklahoma, 1987. 